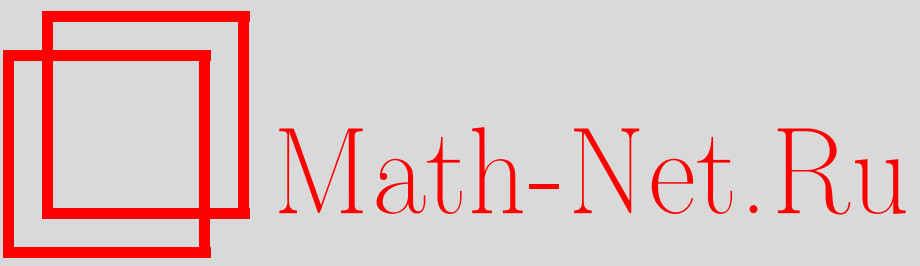

В. В. Жаринов, О дифференцированиях алгебры Гейзенберга, ТМФ, 1999, том 118, номер 2, 163-189

DOI: https://doi.org/10.4213/tmf692

Использование Общероссийского математического портала Math-Net.Ru подразумевает, что вы прочитали и согласны с пользовательским соглашением

http://www . mathnet.ru/rus/agreement

Параметры загрузки:

IP : 54.164 .48 .24

26 апреля 2023 г., $11: 10: 30$ 
ТЕОРЕТИЧЕСКАЯ

И МАТЕМАТИЧЕСКАЯ

ФИЗИКА

Том 118, № 2

февраль, 1999

(C) $\mathbf{1 9 9 9}$ г.

В.В. Жаринов*

\section{О ДИФФЕРЕНЦИРОВАНИЯХ АЛГЕБРЫ ГЕЙЗЕНБЕРГА}

Исследуются дифференцирования алгебры Гейзенберга $\mathcal{H}$ и связанные с ними вопросы. Используются идеи и язык формальной дифференциальной геометрии. Доказано, что все дифференцирования алгебры $\mathcal{H}$ внутренние. Выделены основные подалгебры алгебры Ли $\mathfrak{D}(\mathcal{H})$ всех дифференцирований $\mathcal{H}$ и изучены их свойства. Показано, что сама алгебра $\mathcal{H}$, рассматриваемая как алгебра Ли (с коммутатором в качестве скобки Ли), образует одномерное центральное расширение алгебры $\mathfrak{D}(\mathcal{H})$.

\section{ВВЕДЕНИЕ}

Алгебра Гейзенберга (т.е. алгебра многочленов с образующими, удовлетворяющими квантовым перестановочным соотношениям, см., например, $[1,2])$ - один из фундаментальных объектов квантовой теории, в той или иной форме она присутствует во многих физических и математических моделях и теориях. В последнее время этой алгеброй и ее обобшениями (деформациями) активно интересуются как физики-теоретики, так и математики, рассматривая ее и как фундаментальный объект, и как удобную модель для проверки разнообразных физических и математических идей и конструкций (см., например, [3-8]).

В предлагаемой работе изучаются алгебра Ли всех дифференцирований алгебры Гейзенберга, ее основные подалгебры и возникающие попутно структуры. Желание сделать работу замкнутой привело к тому, что она приобрела отчасти обзорный и методический характер. Так, ради полноты изложения приводятся основы формальной дифференциальной геометрии. С учетом существующих различных подходов к последней (см., например, [9-15]) это представляется разумным (особенно, что касается понятия мультипликатора и его роли в построении теории, см., например, [16]). Многогранность и обыденность использования алгебры Гейзенберга привели к тому, что ее основные свойства известны часто на уровне фольклора, что затрудняет указание точных ссылок на оригинальные работы. Это мотивировало написание раздела с формальным определением алгебры Гейзенберга и перечислением ее элементарных свойств. Отметим, что такое подробное введение позволило сделать дальнейшее изложение ясным и компактным, а также дать представление о месте полученных результатов в контексте общей теории.

* Математический институт им. В. А. Стеклова РАН, Москва, Россия. E-mail: victor@zharinov.mian.su; zharinov@genesis.mi.ras.ru 


\section{0. ЭЛЕМЕНТЫ ФОРМАЛЬНОЙ ДИФФЕРЕНЦИАЛЬНОЙ ГЕОМЕТРИИ}

0.1. Обозначения. Пусть $\mathbb{F}$ - фиксированное поле нулевой характеристики. Пусть $\mathcal{A}$ - топологическая алгебра над $\mathbb{F}$ ( $\mathbb{F}$-алгебра). Обозначим:

cen $\mathcal{A}=\{f \in \mathcal{A}: f g=g f, \forall g \in \mathcal{A}\}-$ иентр $\mathcal{A}$, ann $\mathcal{A}=\{f \in \mathcal{A}: f g=g f=0, \forall g \in \mathcal{A}\}-$ аннулятор $\mathcal{A}$,

$\mathfrak{L}(\mathcal{A})$ - линейное пространство всех линейных непрерывных отображений из $\mathcal{A}$ в $\mathcal{A}$.

Композиция $F, G \mapsto F \circ g$ определяет в $\mathfrak{L}(\mathcal{A})$ структуру унитальной (т.е. содержашей единицу) ассоциативной алгебры $\mathfrak{L}_{0}(\mathcal{A})$, а коммутатор $F, G \mapsto[F, G]=F \circ G-G \circ F$ определяет структуру алгебры Ли $\mathfrak{L}_{[,]}(\mathcal{A})$.

Действие (точнее, левое действие) $\alpha: \mathcal{A} \rightarrow \mathfrak{L}(\mathcal{A})$ определяется правилом $f \mapsto \alpha(f)$, $\alpha(f)(g)=f g, \forall f, g \in \mathcal{A}$.

0.2. Мультипликаторы. Отображение $M \in \mathfrak{L}(\mathcal{A})$ называется мультипликатором (см., например, [16]), если $M(f g)=M(f) g=f M(g), \forall f, g \in \mathcal{A}$.

Множество $\mathfrak{M}(\mathcal{A})$ всех мультипликаторов алгебры $\mathcal{A}$ обладает следуюшими свойствами:

(1) $\mathfrak{M}(\mathcal{A})$ - унитальная подалгебра алгебры $\mathfrak{L}_{0}(\mathcal{A})$;

(2) ядро $\operatorname{ker} M=\{f \in \mathcal{A}: M(f)=0\}$ и образ $\operatorname{im} M=M(\mathcal{A})$ суть двусторонние идеалы алгебры $\mathcal{A}, \forall M \in \mathfrak{M}(\mathcal{A})$;

(3) $M: \operatorname{ann} \mathcal{A} \rightarrow \operatorname{ann} \mathcal{A}$ и $M: \operatorname{cen} \mathcal{A} \rightarrow \operatorname{cen} \mathcal{A}, \forall M \in \mathfrak{M}(\mathcal{A})$;

(4) $[M, N](f) \in \operatorname{ann} \mathcal{A}, \forall M, N \in \mathfrak{M}(\mathcal{A})$ и $\forall f \in \mathcal{A}$; в частности, алгебра $\mathfrak{M}(\mathcal{A})$ коммутативная, если ann $\mathcal{A}=0$.

Если алгебра $\mathcal{A}$ ассоциативная, то дополнительно:

(5) $\alpha: \operatorname{cen} \mathcal{A} \rightarrow \mathfrak{M}(\mathcal{A})$, т.е. действие $\alpha(f) \in \mathfrak{M}(\mathcal{A}), \forall f \in \operatorname{cen} \mathcal{A}$;

(6) пересечение $\operatorname{ker} \alpha \cap \operatorname{cen} \mathcal{A}=\{f \in \operatorname{cen} \mathcal{A}: \alpha(f)=0\}=\operatorname{ann} \mathcal{A}$; в частности, отображение $\alpha: \operatorname{cen} \mathcal{A} \rightarrow \mathfrak{M}(\mathcal{A})$ инъективное, если ann $\mathcal{A}=0$;

(7) если существует аппроксимация единищы $\left\{\delta_{n}\right\} \subset \mathcal{A}$ (так что $\delta_{n} f \rightarrow f$ и $f \delta_{n} \rightarrow f$, $n \rightarrow \infty, \forall f \in \mathcal{A})$, то ann $\mathcal{A}=0$ и образ $\alpha(\operatorname{cen} \mathcal{A})$ плотен в $\mathfrak{M}(\mathcal{A})$.

Наконец, если $\mathcal{A}$ - унитальная ассоциативная алгебра, то

(8) $\alpha$ : $\operatorname{cen} \mathcal{A} \simeq \mathfrak{M}(\mathcal{A})$, обратное отображение $\alpha^{-1}$ действует по правилу $M \mapsto$ $\alpha^{-1}(M)=M(e), \forall M \in \mathfrak{M}(\mathcal{A})$, где $e$ - единища алгебры $\mathcal{A} ;$ в частности, можно отождествить алгебры $\mathfrak{M}(\mathcal{A})$ и сеп $\mathcal{A}$.

0.3. Дифференцирования. Отображение $D \in \mathfrak{L}(\mathcal{A})$ называется дифферениированием, если выполняется правило Лейбница: $D(f g)=D(f) g+f D(g), \forall f, g \in \mathcal{A}$. 
Множество $\mathfrak{D}(\mathcal{A})$ всех дифференцирований алгебры $\mathcal{A}$ обладает следующими свойствами:

(1) $\mathfrak{D}(\mathcal{A})$ есть левый $\mathfrak{M}(\mathcal{A})$-модуль, $M D=M \circ D, \forall M \in \mathfrak{M}(\mathcal{A})$ и $\forall D \in \mathfrak{D}(\mathcal{A})$;

$(2) \mathfrak{D}(\mathcal{A})$ есть подалгебра алгебры Ли $\mathfrak{L}_{[,]}(\mathcal{A})$;

(3) $D: \operatorname{ann} \mathcal{A} \rightarrow \operatorname{ann} \mathcal{A}$ и $D: \operatorname{cen} \mathcal{A} \rightarrow \operatorname{cen} \mathcal{A}, \forall D \in \mathfrak{D}(\mathcal{A})$;

(4) коммутатор $[D, M] \in \mathfrak{M}(\mathcal{A}), \forall D \in \mathfrak{D}(\mathcal{A})$ и $\forall M \in \mathfrak{M}(\mathcal{A})$.

В частности, для каждого $D \in \mathfrak{D}(\mathcal{A})$ правило $\widehat{D}(M)=[D, M], \quad M \in \mathfrak{M}(\mathcal{A})$, задает отображение $\widehat{D}: \mathfrak{M}(\mathcal{A}) \rightarrow \mathfrak{M}(\mathcal{A})$. Более того, в силу тождества Якоби

(5) $\widehat{D}(M \circ N)=\widehat{D}(M) \circ N+M \circ \widehat{D}(N), \forall D \in \mathfrak{D}(\mathcal{A})$ и $\forall M, N \in \mathfrak{M}(\mathcal{A})$.

Таким образом, правило $D \mapsto \beta(D)=\widehat{D}, D \in \mathfrak{D}(\mathcal{A})$, определяет действие $\beta: \mathfrak{D}(\mathcal{A}) \rightarrow$ $\mathfrak{D}(\mathfrak{M}(\mathcal{A}))$.

(6) Действие $\beta: \mathfrak{D}(\mathcal{A}) \rightarrow \mathfrak{D}(\mathfrak{M}(\mathcal{A}))$ есть морфизм алгебр Ли.

(7) Справедливо расширенное правило Лейбница: $D(M(f))=\widehat{D}(M)(f)+M(D(f))$, $\forall D \in \mathfrak{D}(\mathcal{A}), \forall M \in \mathfrak{M}(\mathcal{A})$ и $\forall f \in \mathcal{A}$.

Если алгебра $\mathcal{A}$ ассоциативная, то

(8) действия $\alpha$ на сеп $\mathcal{A}$ и $\beta$ на $\mathfrak{D}(\mathcal{A})$ совместны, т.е. $\widehat{D}(\alpha(f))=\alpha(D(f)), \forall D \in \mathfrak{D}(\mathcal{A})$ и $\forall f \in \operatorname{cen} \mathcal{A}$;

$(9)$ для каждого $f \in \mathcal{A}$ действие $\alpha_{-}(f): \mathcal{A} \rightarrow \mathcal{A}, \alpha_{-}(f)(g)=[f, g], g \in \mathcal{A}$, является дифференцированием алгебры $\mathcal{A}\left(\right.$ т.е. $\left.\alpha_{-}(f) \in \mathfrak{D}(\mathcal{A})\right)$.

Дифференцирование вида $\alpha_{-}(f)$ называется внутренним, очевидно, оно нетривиальное, если $f \notin \operatorname{cen} \mathcal{A}$.

0.4. Константы. Элемент $f \in \mathcal{A}$ называется константой, если $D(f)=0, \forall D \in$ $\mathfrak{D}(\mathcal{A})$. Обозначим con $\mathcal{A}$ множество всех констант алгебры $\mathcal{A}$. Очевидно, что $\operatorname{con} \mathcal{A}$ есть подалгебра алгебры $\mathcal{A}$, причем если алгебра $\mathcal{A}$ унитальна, то ее единица $e \in \operatorname{con} \mathcal{A}$.

Подмножество $\mathcal{S} \subset \mathfrak{D}(\mathcal{A})$ называется тотальныцм. если условие $D(f)=0, \forall D \in \mathcal{S}$, влечет $f \in \operatorname{con} \mathcal{A}$.

0.5. Дифференциальные алгебры. Дифференциальная алгебра есть пара $(\mathcal{A}, \mathfrak{C}(\mathcal{A}))$, где $\mathcal{A}$ - алгебра, а $\mathfrak{C}(\mathcal{A})$ - подалгебра алгебры Ли $\mathfrak{D}(\mathcal{A})$ (см., например, $[17,18])$. Дифференциальная алгебра $(\mathcal{A}, \mathfrak{C}(\mathcal{A}))$ называется стандартной, если $\mathfrak{C}(\mathcal{A})$ есть свободный конечномерный подмодуль левого $\mathfrak{M}(\mathcal{A})$-модуля $\mathfrak{D}(\mathcal{A})$ с попарно коммутируюшим базисом $D_{1}, \ldots, D_{m} \in \mathfrak{D}(\mathcal{A})\left(\left[D_{\mu}, D_{\nu}\right]=0, \mu, \nu \in \overline{1, m}\right)$, так что

$$
\mathfrak{C}(\mathcal{A}) \equiv[D]=\left\{D=\sum_{\mu=1}^{m} M_{\mu} D_{\mu}: M_{\mu} \in \mathfrak{M}(\mathcal{A}), \mu \in \overline{1, m}\right\}
$$

(здесь и всюду ниже $\overline{k, l} \equiv\{k, k+1, \ldots, l\}$ ). Дифференциальные алгебры возникают в алгебро-геометрическом подходе к дифференциальным уравнениям. Учитывая это, мы 
будем использовать принятую там терминологию (см., например, [19, 20]). Так, дифференцирования из подалгебры $\mathfrak{C}(\mathcal{A})$ называются картановы.ми .

Дифференцирование $X \in \mathfrak{D}(\mathcal{A})$ называется дифференцированием Ли-Беклунда, если $[X, D] \in \mathfrak{C}(\mathcal{A}), \forall D \in \mathfrak{C}(\mathcal{A})$. Множество всех таких дифференцирований образует подалгебру $\mathfrak{B}(\mathcal{A})$ алгебры Ли $\mathfrak{D}(\mathcal{A})$, причем по построению $\mathfrak{C}(\mathcal{A})$ есть идеал в $\mathfrak{B}(\mathcal{A})$. Фактор-алгебра $\operatorname{Sym}_{\mathfrak{C}(\mathcal{A})} \mathcal{A}=\mathfrak{B}(\mathcal{A}) / \mathfrak{C}(\mathcal{A})$ называется алгеброй Ли симметрий дифференциальной алгебры $(\mathcal{A}, \mathfrak{C}(\mathcal{A}))$.

0.6. Дифференциальные формы. Будем считать, что изучаемая алгебра $\mathcal{A}$ удовлетворяет условию:

(i) алгебра мультипликаторов $\mathfrak{M}(\mathcal{A})$ коммутативная (например, ann $\mathcal{A}=0$ ).

В этом случае $\mathfrak{D}(\mathcal{A})$ есть $\mathfrak{M}(\mathcal{A})$-модуль, и определена его внешняя алгебра

$$
\wedge^{*} \mathfrak{D}(\mathcal{A})=\prod_{r=0}^{\infty} \wedge^{r} \mathfrak{D}(\mathcal{A}), \quad \wedge^{0} \mathfrak{D}(\mathcal{A})=\mathfrak{M}(\mathcal{A}), \quad \wedge^{1} \mathfrak{D}(\mathcal{A})=\mathfrak{D}(\mathcal{A}), \quad \ldots
$$

В частности, для любых $D_{1}, \ldots, D_{r} \in \mathfrak{D}(\mathcal{A})$ внешнее произведение

$$
D_{1} \wedge \cdots \wedge D_{r}=\frac{1}{r !} \sum_{\pi \in \mathfrak{S}_{r}} \operatorname{sign} \pi D_{\pi(1)} \otimes \cdots \otimes D_{\pi(r)} \in \wedge^{r} \mathfrak{D}(\mathcal{A}),
$$

где $\mathfrak{S}_{r}$ - группа всех перестановок чисел $1, \ldots, r$.

Пусть $\mathcal{K}$ - произвольная $\mathbb{F}$-алгебра такая, что:

(ii) определен морфизм ассоциативных алгебр $\varphi: \mathfrak{M}(\mathcal{A}) \rightarrow \mathfrak{M}(\mathcal{K})$,

(iii) определен морфизм алгебр Ли $\psi: \mathfrak{D}(\mathcal{A}) \rightarrow \mathfrak{D}(\mathcal{K})$,

(iv) выполнены условия согласования

$\psi(M D)=\varphi(M) \psi(D) \quad$ и $\varphi([M, D])=[\varphi(M), \psi(D)], \quad \forall M \in \mathfrak{M}(\mathcal{A}) \quad$ и $\quad D \in \mathfrak{D}(\mathcal{A})$

$($ Напомним, что $M D \in \mathfrak{D}(\mathcal{A})$ и $[M, D] \in \mathfrak{M}(\mathcal{A})$.) $\quad$ В частности, алгебра $\mathcal{K}$ есть $\mathfrak{M}(\mathcal{A})$-модуль.

ЗАмЕЧАнИЕ. Условиям (ii)-(iv) удовлетворяют, например, следуюшие алгебры (считаем, что условие (i) выполнено):

(1) сама алгебра $\mathcal{A}$, в этом случае $\varphi=\operatorname{id}_{\mathfrak{M}(\mathcal{A})}$ и $\psi=\operatorname{id}_{\mathfrak{D}(\mathcal{A})}$;

(2) алгебра мультипликаторов $\mathfrak{M}(\mathcal{A})$, в этом случае $\varphi=\mathrm{id}_{\mathfrak{M}(\mathcal{A})}$ (поясним, что в силу (i) $\mathfrak{M}(\mathfrak{M}(\mathcal{A})) \cong \mathfrak{M}(\mathcal{A}))$, а морфизм $\psi: \mathfrak{D}(\mathcal{A}) \rightarrow \mathfrak{D}(\mathfrak{M}(\mathcal{A}))$ определяется правилом $\psi(D)(M)=[D, M], \forall D \in \mathfrak{D}(\mathcal{A}), M \in \mathfrak{M}(\mathcal{A})$;

$(3)$ алгебра Ли дифференцирований $\mathfrak{D}(\mathcal{A})$ при условии $[\mathfrak{M}(\mathcal{A}), \mathfrak{D}(\mathcal{A})]=0$, здесь морфизм $\varphi: \mathfrak{M}(\mathcal{A}) \rightarrow \mathfrak{M}(\mathfrak{D}(\mathcal{A}))$ дается правилом $\varphi(M)(X)=M X$, а морфизм $\psi:$ $\mathfrak{D}(\mathcal{A}) \rightarrow \mathfrak{D}(\mathfrak{D}(\mathcal{A}))$ - правилом $\psi(D)(X)=[D, X], \forall M \in \mathfrak{M}(\mathcal{A})$ и $D, X \in \mathfrak{D}(\mathcal{A})$. 
Отметим, что условие $[\mathfrak{M}(\mathcal{A}), \mathfrak{D}(\mathcal{A})]=0$ выполняется, если $\mathfrak{M}(\mathcal{A})=\mathbb{F} \cdot \operatorname{id} \mathcal{A}_{\mathcal{A}}$ очевидно, что в этом случае условие (i) также выполнено.

Положим

$$
\Omega^{*}(\mathcal{A} ; \mathcal{K})=\operatorname{Hom}_{\mathfrak{M}(\mathcal{A})}\left(\wedge^{*} \mathfrak{D}(\mathcal{A}) ; \mathcal{K}\right)=\sum_{r=0}^{\infty} \Omega^{r}(\mathcal{A} ; \mathcal{K})
$$

Подробнее,

$$
\Omega^{0}(\mathcal{A} ; \mathcal{K})=\operatorname{Hom}_{\mathfrak{M}(\mathcal{A})}(\mathfrak{M}(\mathcal{A}) ; \mathcal{K}) \cong \mathcal{K}
$$

(изоморфизм задается правилом $\left.\Omega^{0}(\mathcal{A} ; \mathcal{K}) \ni \omega \mapsto k=\omega\left(\operatorname{id}_{\mathcal{A}}\right) \in \mathcal{K}\right)$, а при $r>0$

$$
\omega\left(D_{1}, \ldots, D_{r}\right) \equiv \omega\left(D_{1} \wedge \cdots \wedge D_{r}\right), \quad \omega \in \Omega^{r}(\mathcal{A} ; \mathcal{K})
$$

есть кососимметрическая функция, $\mathfrak{M}(\mathcal{A})$-линейная по каждому из аргументов $D_{1}, \ldots, D_{r} \in \mathfrak{D}(\mathcal{A})$. Элементы из $\Omega^{r}(\mathcal{A} ; \mathcal{K})$ называются дифференциальнылми $r$-формами.

0.7. Операции над дифференциальными формами. Перечислим основные структуры, определенные на $\Omega^{*}(\mathcal{A} ; \mathcal{K})$, и их свойства. Прежде всего отметим, что $\Omega^{*}(\mathcal{A} ; \mathcal{K})$ есть $\mathfrak{M}(\mathcal{A})$-модуль, причем по определению

$$
(M \omega)\left(D_{1}, \ldots, D_{r}\right)=\varphi(M)\left(\omega\left(D_{1}, \ldots, D_{r}\right)\right)
$$

для всех $M \in \mathfrak{M}(\mathcal{A}), \omega \in \Omega^{r}(\mathcal{A} ; \mathcal{K})$ и $D_{1}, \ldots, D_{r} \in \mathfrak{D}(\mathcal{A})$.

Линейные операции (считаем $\Omega^{r}(\mathcal{A} ; \mathcal{K}=0)$ при $r<0$ и $\left.D \in \mathfrak{D}(\mathcal{A})\right)$ :

$$
\begin{aligned}
& \text { свертка } \quad \iota_{D}: \Omega^{r}(\mathcal{A} ; \mathcal{K}) \rightarrow \Omega^{r-1}(\mathcal{A} ; \mathcal{K}), \\
& \text { производная Ли } L_{D}: \Omega^{r}(\mathcal{A} ; \mathcal{K}) \rightarrow \Omega^{r}(\mathcal{A} ; \mathcal{K}) \text {, } \\
& \text { диффференциал } \quad d: \Omega^{r}(\mathcal{A} ; \mathcal{K}) \rightarrow \Omega^{r+1}(\mathcal{A} ; \mathcal{K}) \text {, }
\end{aligned}
$$

зададим соответственно формулами (считаем $\omega \in \Omega^{r}(\mathcal{A} ; \mathcal{K})$ )

$$
\left(\iota_{D} \omega\right)\left(D_{1}, \ldots, D_{r-1}\right)=\omega\left(D, D_{1}, \ldots, D_{r-1}\right), \quad D_{1}, \ldots, D_{r-1} \in \mathfrak{D}(\mathcal{A})
$$

(в частности, $\iota_{D} \omega=0$ при $r=0$ );

$$
\left(L_{D} \omega\right)\left(D_{1}, \ldots, D_{r}\right)=\psi(D)\left(\omega\left(D_{1}, \ldots, D_{r}\right)\right)-\sum_{i=1}^{r} \omega\left(D_{1}, \ldots,\left[D, D_{i}\right], \ldots, D_{r}\right)
$$

для $D_{1}, \ldots, D_{r} \in \mathfrak{D}(\mathcal{A})$ и, наконец,

$$
\begin{aligned}
(d \omega)\left(D_{0}, \ldots, D_{r}\right)= & \sum_{i=0}^{r}(-1)^{i} \psi\left(D_{i}\right)\left(\omega\left(D_{0}, \ldots, \check{D}_{i}, \ldots, D_{r}\right)\right)+ \\
& +\sum_{0 \leqslant i<j \leqslant r}(-1)^{i+j} \omega\left(\left[D_{i}, D_{j}\right], D_{0}, \ldots, \check{D}_{i}, \ldots, \check{D}_{j}, \ldots, D_{r}\right)
\end{aligned}
$$


для $D_{0}, \ldots, D_{r} \in \mathfrak{D}(\mathcal{A})$, значок`над какой-либо переменной означает, что эта переменная опушена.

Введенные операции обладают стандартными свойствами:

(1) $\iota_{X} \circ \iota_{Y}+\iota_{Y} \circ \iota_{X}=0, \quad \forall X, Y \in \mathfrak{D}(\mathcal{A})$;

(2) $L_{X} \circ L_{Y}-L_{Y} \circ L_{X}=L_{[X, Y]}, \quad \forall X, Y \in \mathfrak{D}(\mathcal{A})$;

(3) $d \circ d=0$;

(4) $L_{D}=d \circ \iota_{D}+\iota_{D} \circ d, \quad \forall D \in \mathfrak{D}(\mathcal{A})$;

(5) $L_{D} \circ d=d \circ L_{D}, \quad \forall D \in \mathfrak{D}(\mathcal{A})$.

Корректность приведенных определений и справедливость соотношений (1)-(5) проверяется с помощью условий (i)-(iv) раздела 0.6.

0.8. Алгебра дифференциальных форм. Определим в $\Omega^{*}(\mathcal{A} ; \mathcal{K})$ умножсение правилом

$$
\omega \in \Omega^{r}(\mathcal{A} ; \mathcal{K}), \quad \theta \in \Omega^{s}(\mathcal{A} ; \mathcal{K}) \mapsto \omega \cdot \theta \in \Omega^{r+s}(\mathcal{A} ; \mathcal{K})
$$

где

$$
\begin{aligned}
& (\omega \cdot \theta)\left(D_{1}, \ldots, D_{r+s}\right)= \\
& \quad=\frac{1}{(r+s) !} \sum_{\pi \in \mathfrak{S}_{r+s}} \operatorname{sign} \pi \omega\left(D_{\pi(1)}, \ldots, D_{\pi(r)}\right) \theta\left(D_{\pi(r+1)}, \ldots, D_{\pi(r+s)}\right)
\end{aligned}
$$

для всех $D_{1}, \ldots, D_{r+s} \in \mathfrak{D}(\mathcal{A})$.

Это умножение обладает следуюшими свойствами (считаем $\omega \in \Omega^{r}(\mathcal{A} ; \mathcal{K}), \quad \theta \in$ $\left.\Omega^{s}(\mathcal{A} ; \mathcal{K})\right):$

(1) $\iota_{D}(\omega \cdot \theta)=\left(\iota_{D} \omega\right) \cdot \theta+(-1)^{r} \omega \cdot\left(\iota_{D} \theta\right), \forall D \in \mathfrak{D}(\mathcal{A})$;

(2) $L_{D}(\omega \cdot \theta)=\left(L_{D} \omega\right) \cdot \theta+\omega \cdot\left(L_{D} \theta\right), \forall D \in \mathfrak{D}(\mathcal{A})$;

(3) $d(\omega \cdot \theta)=(d \omega) \cdot \theta+(-1)^{r} \omega \cdot(d \theta)$;

(4) если алгебра $\mathcal{K}$ коммутативная, то $\omega \cdot \theta=(-1)^{r s} \theta \cdot \omega$.

Корректность определения операции умножения и справедливость формул (1)-(4) проверяется с помошью условий (i)-(iv) раздела 0.6 .

0.9. Когомологии де Рама. В силу пункта (3) раздела 0.7 определен комплекс линейных пространств

$$
0 \rightarrow \Omega^{0}(\mathcal{A} ; \mathcal{K}) \stackrel{d}{\rightarrow} \Omega^{1}(\mathcal{A} ; \mathcal{K}) \stackrel{d}{\rightarrow} \cdots \stackrel{d}{\rightarrow} \Omega^{r}(\mathcal{A} ; \mathcal{K}) \stackrel{d}{\rightarrow} \Omega^{r+1}(\mathcal{A} ; \mathcal{K}) \stackrel{d}{\rightarrow} \cdots,
$$

его когомологии $H^{r}(\mathcal{A} ; \mathcal{K}), r=0,1, \ldots$, называются когомологиями де Рама алгебрьи $\mathcal{A}$ со значениями в алгебре $\mathcal{K}$. Например,

$$
H^{0}(\mathcal{A} ; \mathcal{K})=\{\omega \in \mathcal{K}: \psi(D)(\omega)=0, \quad \forall D \in \mathfrak{D}(\mathcal{A})\}
$$

Положим

$$
H^{*}(\mathcal{A} ; \mathcal{K})=\sum_{r=0}^{\infty} H^{r}(\mathcal{A} ; \mathcal{K})
$$

Правило

$$
[\omega] \in H^{r}(\mathcal{A} ; \mathcal{K}), \quad[\theta] \in H^{s}(\mathcal{A} ; \mathcal{K}) \mapsto[\omega] \cdot[\theta]=[\omega \cdot \theta] \in H^{r+s}(\mathcal{A} ; \mathcal{K})
$$

определяет в линейном пространстве $H^{*}(\mathcal{A} ; \mathcal{K})$ умножение и преврашает его в $\mathbb{F}$-алгебру. 
0.10. Картановы формы. Пусть $(\mathcal{A}, \mathfrak{C}(\mathcal{A}))$ - дифференциальная алгебра (см. раздел 0.5$), \mathcal{K}$ - некоторая $\mathbb{F}$-алгебра, и по-прежнему выполнены условия (i)-(iv) раздела 0.6 .

Дифференциальная $r$-форма $\omega \in \Omega^{r}(\mathcal{A} ; \mathcal{K})$ называется $s$-картановой, если $\omega\left(D_{1}, \ldots, D_{r}\right)=0$ всякий раз, когда среди дифференцирований $D_{1}, \ldots, D_{r}$ имеются хотя бы $r-s+1$ картановых. $\mathfrak{M}(\mathcal{A})$-модуль всех $s$-картановых $r$-форм обозначим через $C^{s} \Omega^{r}(\mathcal{A} ; \mathcal{K})$.

Приведем основные свойства операций над картановыми формами (по определению $C^{s} \Omega^{r}(\mathcal{A} ; \mathcal{K})=\Omega^{r}(\mathcal{A} ; \mathcal{K})$ при $s<0$ и $C^{s} \Omega^{r}(\mathcal{A} ; \mathcal{K})=0$ при $s>r$ или $\left.r<0\right)$ :

(1) $\omega \cdot \theta \in C^{s+q} \Omega^{r+p}(\mathcal{A} ; \mathcal{K}), \forall \omega \in C^{s} \Omega^{r}(\mathcal{A} ; \mathcal{K}), \theta \in C^{q} \Omega^{p}(\mathcal{A} ; \mathcal{K})$;

(2) $\iota_{D}: C^{s} \Omega^{r}(\mathcal{A} ; \mathcal{K}) \rightarrow C^{s-1} \Omega^{r-1}(\mathcal{A} ; \mathcal{K}), \quad \forall D \in \mathfrak{D}(\mathcal{A})$;

(3) $\iota_{D}: C^{s} \Omega^{r}(\mathcal{A} ; \mathcal{K}) \rightarrow C^{s} \Omega^{r-1}(\mathcal{A} ; \mathcal{K}), \forall D \in \mathfrak{C}(\mathcal{A})$;

(4) $L_{D}: C^{s} \Omega^{r}(\mathcal{A} ; \mathcal{K}) \rightarrow C^{s-1} \Omega^{r}(\mathcal{A} ; \mathcal{K}), \forall D \in \mathfrak{D}(\mathcal{A})$;

(5) $L_{D}: C^{s} \Omega^{r}(\mathcal{A} ; \mathcal{K}) \rightarrow C^{s} \Omega^{r}(\mathcal{A} ; \mathcal{K}), \forall D \in \mathfrak{B}(\mathcal{A})$

(6) $d: C^{s} \Omega^{r}(\mathcal{A} ; \mathcal{K}) \rightarrow C^{s} \Omega^{r+1}(\mathcal{A} ; \mathcal{K})$.

0.11. Спектральная последовательность. Сохраняем обозначения раздела 0.10 и добавляем сокрашение $C^{s} \Omega^{r} \equiv C^{s} \Omega^{r}(\mathcal{A} ; \mathcal{K})$. По построению имеется фильтрация

$$
\Omega^{r}=C^{0} \Omega^{r} \supset C^{1} \Omega^{r} \supset C^{2} \Omega^{r} \supset \cdots \supset C^{r} \Omega^{r} \supset 0,
$$

совместимая с градуировкой

$$
\Omega^{*}=\sum_{r=0}^{\infty} \Omega^{r}
$$

и дифференциалом $d: \Omega^{r} \rightarrow \Omega^{r+1}, r=0,1, \ldots$ Согласно общей теории (см., например, [21]) определена спектральная последовательность $\left\{E_{r}^{p, q}, d_{r}^{p, q} ; p, q, r=0,1, \ldots\right\}$ с членами

$$
E_{r}^{p, q} \equiv E_{r}^{p, q}(\mathcal{A}, \mathfrak{C}(\mathcal{A}) ; \mathcal{K})=\frac{Z_{r}^{p, q}}{B_{r-1}^{p, q}+Z_{r-1}^{p+1, q-1}}
$$

где

$$
\begin{aligned}
& Z_{r}^{p, q}=\left\{\omega \in C^{p} \Omega^{p+q}: d \omega \in C^{p+r} \Omega^{p+q+1}\right\}, \\
& B_{r}^{p, q}=\left\{\omega=d \theta \in C^{p} \Omega^{p+q}: \theta \in C^{p-r} \Omega^{p+q-1}\right\},
\end{aligned}
$$

а линейные отображения

$$
d_{r}^{p, q}: E_{r}^{p, q} \rightarrow E_{r}^{p+r, q-r+1}
$$

индуцированы дифференциалом $d$ (т.е. $d_{r}^{p, q}[\omega]=[d \omega], \forall[\omega] \in E_{r}^{p, q}$ ). Члены порядка $r=\infty$ определяются формулами

$$
E_{\infty}^{p, q}=\frac{Z_{\infty}^{p, q}}{B_{\infty}^{p, q}+Z_{\infty}^{p+1, q-1}}, \quad p, q=0,1, \ldots,
$$

где

$$
\begin{aligned}
& Z_{\infty}^{p, q}=\left\{\omega \in C^{p} \Omega^{p+q}: d \omega=0\right\}, \\
& B_{\infty}^{p, q}=\left\{\omega=d \theta \in C^{p} \Omega^{p+q}: \theta \in \Omega^{p+q-1}\right\} .
\end{aligned}
$$


Перечислим основные свойства спектральной последовательности:

(1) $d_{r}^{p+r, q-r+1} \circ d_{r}^{p, q}=0, E_{r+1}^{p, q}=\operatorname{ker} d_{r}^{p, q} / \operatorname{im} d_{r}^{p-r, q+r-1}$;

(2) $E_{\infty}^{p, q}=E_{r}^{p, q}, r>\max \{p, q+1\}$.

Пусть симметрия $[D] \in \operatorname{Sym} \mathcal{A}$ (см. раздел 0.5$)$,

(3) линейное отображение $\iota_{[D]}: E_{r}^{p, q} \rightarrow E_{r}^{p-r, q+r-1}$ определено правилом $\iota_{[D]}[\omega]=$ $\left[\iota_{D} \omega\right],[\omega] \in E_{r}^{p, q}$

(4) линейное отображение $L_{[D]}: E_{r}^{p, q} \rightarrow E_{r}^{p, q}$ определено аналогичньм правилом $L_{[D]}[\omega]=\left[L_{D} \omega\right],[\omega] \in E_{r}^{p, q}$

(5) $L_{[D]} \circ d_{r}^{p, q}=d_{r}^{p, q} \circ L_{[D]}$;

(6) $L_{[D]}=\iota_{[D]} \circ d_{r}^{p, q}+d_{r}^{p, q} \circ \iota_{[D]}$.

0.12. Связь с когомологиями де Рама. Сохраняем соглашения раздела 0.11 и добавим сокрашение $H^{k} \equiv H^{k}(\mathcal{A} ; \mathcal{K})$. Фильтрация дифференшиальных форм картановыми формами (раздела 0.11) порождает фильтрацию когомологий де Рама

$$
H^{k}=H^{0, k} \supset H^{1, k} \supset H^{2, k} \supset \cdots \supset H^{k, k} \supset 0, \quad k=0,1, \ldots,
$$

где линейные пространства

$$
H^{p, k}=\frac{\left\{\omega \in C^{p} \Omega^{k}: d \omega=0\right\}}{\left\{\omega=d \theta \in C^{p} \Omega^{k}: \theta \in \Omega^{k-1}\right\}}, \quad p, k=0,1, \ldots .
$$

(1) Справедливы равенства $E_{\infty}^{p, k-p}=H^{p, k} / H^{p+1, k}, p, k=0,1, \ldots$, другими словами, имеется равенство градуированных линейных пространств $E_{\infty}^{k}=G\left(H^{k}\right)$, $k=0,1, \ldots$, где по определению

$$
E_{\infty}^{k}=\sum_{p+q=k} E_{\infty}^{p, q}
$$

a

$$
G\left(H^{k}\right)=\sum_{p=0}^{k} H^{p, k} / H^{p+1, k}
$$

- градуированное пространство, ассоциированное с введенной вьше фильтрацией.

\section{1. АЛГЕБРА ГЕЙЗЕНБЕРГА}

1.1. Обозначения. Будем использовать мультииндексные обозначения:

$\mathbb{Z}=\{0, \pm 1, \pm 2, \ldots\} \supset \mathbb{Z}_{+}=\{0,1,2, \ldots\} \supset \mathbb{N}=\{1,2, \ldots\} ;$

$i=\left(i_{1}, \ldots, i_{d}\right) \in \mathbb{Z}^{d}, d \in \mathbb{N}$ фиксировано;

$|i|=i_{1}+\cdots+i_{d}, i \pm j=\left(i_{1} \pm j_{1}, \ldots, i_{d} \pm j_{d}\right), i, j \in \mathbb{Z}$;

$(\mu)=(0, \ldots, 1, \ldots, 0) \in \mathbb{Z}_{+}^{d}$, единица на $\mu$-м месте, $\mu \in \overline{1, d} \equiv\{1, \ldots, d\} ;$

$i \leqslant j, i, j \in \mathbb{Z}^{d}$, если $i_{\mu} \leqslant j_{\mu}$ для всех $\mu \in \overline{1, d}$; 


$$
\begin{aligned}
i !=i_{1} ! \ldots i_{d} !, i \in \mathbb{Z}^{d}(\iota ! & =\infty \text { при } \iota \in\{-1,-2, \ldots\}) ; \\
\left(\begin{array}{c}
i \\
j
\end{array}\right) & =\frac{i !}{j !(i-j) !}, \quad i \in \mathbb{Z}_{+}^{d}, \quad j \in \mathbb{Z}^{d} ; \\
\left(\begin{array}{c}
i, j \\
k
\end{array}\right) & =k !\left(\begin{array}{c}
i \\
k
\end{array}\right)\left(\begin{array}{c}
j \\
k
\end{array}\right), \quad i, j, k \in \mathbb{Z}_{+}^{d} ; \\
\left(\begin{array}{c}
i, j ; k, l \\
m
\end{array}\right) & =\left(\begin{array}{c}
j, k \\
m
\end{array}\right)-\left(\begin{array}{c}
l, i \\
m
\end{array}\right), \quad i, j, k, l, m \in \mathbb{Z}_{+}^{d} .
\end{aligned}
$$

1.2. ОПРЕДЕЛЕниЕ. Будем считать, что фиксированы поле $\mathbb{F}$ нулевой характеристики $(\mathbb{F}=\mathbb{R}, \mathbb{C}, \ldots)$, размерность $d \in \mathbb{N}$ и константа $\varkappa \in \mathbb{F}, \varkappa \neq 0$.

Пусть $\mathbb{F}\langle r\rangle$ - алгебра некоммутативных многочленов от образуюших $r=(q, p)$, $q=\left(q_{1}, \ldots, q_{d}\right), p=\left(p_{1}, \ldots, p_{d}\right)$ с элементами $f(r)=\sum_{A} c_{A} r^{A}$, где

$$
\begin{aligned}
r_{\alpha} & =q_{\alpha} \text { при } \alpha \in \overline{1, d}, \quad r_{\alpha}=p_{\alpha-d} \text { при } \alpha \in \overline{d+1,2 d} ; \\
A & =\left(\alpha_{1}, \ldots, \alpha_{N}\right), \quad \alpha_{\nu} \in \overline{1,2 d}, \quad \nu \in \overline{1, N}, \quad N=|A| ; \\
c_{A} & =c_{\alpha_{1} \ldots \alpha_{N}} \in \mathbb{F}, \quad \text { лишь конечное число } c_{A} \neq 0 ; \\
r^{A} & =r_{\alpha_{1}} \ldots r_{\alpha_{N}} .
\end{aligned}
$$

Определяющие соотношения (квантовые перестановочные соотношения):

$$
q_{\mu} q_{\nu}-q_{\nu} q_{\mu}=0, \quad p_{\mu} p_{\nu}-p_{\nu} p_{\mu}=0, \quad p_{\mu} q_{\nu}-q_{\nu} p_{\mu}-\varkappa \delta_{\mu \nu}=0, \quad \mu, \nu \in \overline{1, d},
$$

порождают двусторонний идеал $\mathcal{J}$ алгебры $\mathbb{F}\langle r\rangle$. Алгебра Гейзенберга определяется как фактор-алгебра $\mathcal{H}=\mathbb{F}\langle r\rangle / \mathcal{J}$.

1.3. Нормальные многочлены. Многочлен $f \in \mathbb{F}\langle r\rangle$ называется нормальныцм, если

$$
f=f(q, p)=\sum_{i, j} f_{i j} q^{i} p^{j}
$$

где лишь конечное число коэффициентов $f_{i j} \in \mathbb{F}$ ненулевые, $q^{i}=\left(q_{1}\right)^{i_{1}} \ldots\left(q_{d}\right)^{i_{d}}, p_{j}=$ $\left(p_{1}\right)^{j_{1}} \ldots\left(p_{d}\right)^{j_{d}}, i, j \in \mathbb{Z}_{+}^{d}$.

1.4. ПРЕДЛОЖЕНИЕ. Справедливы равенства

$$
p^{j} q^{i}=\sum_{k} \varkappa^{|k|}\left(\begin{array}{c}
j, i \\
k
\end{array}\right) q^{i-k} p^{j-k} \quad(\bmod \mathcal{J}), \quad j, i \in \mathbb{Z}_{+}^{d} .
$$

ДокаЗАТЕЛЬСтво. Пусть сначала $d=1$, так что $i, j, k \in \mathbb{Z}_{+}$. Вычисления проводим по $\bmod \mathcal{J}$, т.е. пренебрегая слагаемыми, лежашими в идеале $\mathcal{J}$. Равенства тривиально справедливы при $i=j=0$, при $i=0, j>0$ и при $i>0, j=0$. Легко также проверить, что

$$
p q^{i}=q^{i} p+i \varkappa q^{i-1} \quad \text { и } p^{j} q=q p^{j}+j \varkappa p^{j-1}, \quad i, j \in \mathbb{N} .
$$


Предположим, что равенства справедливы при некоторых $i, j>0$. Тогда

$$
\begin{aligned}
p^{j+1} q^{i} & =p\left(p^{j} q^{i}\right)=p \sum_{k} \varkappa^{k}\left(\begin{array}{c}
j, i \\
k
\end{array}\right) q^{i-k} p^{j-k}=\sum_{k} \varkappa^{k}\left(\begin{array}{c}
j, i \\
k
\end{array}\right)\left(p q^{i-k}\right) p^{j-k}= \\
& =\sum_{k} \varkappa^{k}\left(\begin{array}{c}
c, i \\
k
\end{array}\right)\left(q^{i-k} p+(i-k) \varkappa q^{i-k-1}\right) p^{j-k}= \\
& =\sum_{k} \varkappa^{k}\left(\begin{array}{c}
j, i \\
k
\end{array}\right) q^{i-k} p^{j+1-k}+\sum_{k} \varkappa^{k+1}\left(\begin{array}{c}
j, i \\
k
\end{array}\right)(i-k) q^{i-k-1} p^{j-k}= \\
& =\sum_{k} \varkappa\left[\left(\begin{array}{c}
j, i \\
k
\end{array}\right)+(i+1-k)\left(\begin{array}{c}
j, i \\
k-1
\end{array}\right)\right] q^{i-k} p^{j+1-k}=\sum_{k} \varkappa^{k}\left(\begin{array}{c}
j+1, i \\
k
\end{array}\right) q^{i-k} p^{j+1-k},
\end{aligned}
$$

поскольку

$$
\left(\begin{array}{c}
j, i \\
k
\end{array}\right)+(i+1-k)\left(\begin{array}{c}
j, i \\
k-1
\end{array}\right)=\left(\begin{array}{c}
j+1, i \\
k
\end{array}\right) .
$$

Аналогично проверяется переход $j, i \mapsto j, i+1$. Итак, в случае $d=1$ равенства верны.

Случай $d>1$ сводится к одномерному с помощью коммутационных соотношений $p_{\mu} q_{\nu}=q_{\nu} p_{\mu}$ при $\mu \neq \nu$.

1.5. СЛЕДСТВИЕ. Каждый многочлен $f \in \mathbb{F}\langle r\rangle$ имеет представление $f(r)=$ $g(q, p)+h(r)$, где $g(q, p)-$ нормальный многочлен, $а$ $h(r) \in \mathcal{J}$.

1.6. Операторное представление. Пусть $\mathbb{F}[x]$ - алгебра коммутативных многочленов от $x=\left(x_{1}, \ldots, x_{d}\right) \in \mathbb{F}^{d}, \mathfrak{L}(\mathbb{F}[x])$ - алгебра непрерывных линейных отображений $\mathbb{F}[x]$ в себя. Правило $q_{\mu}, p_{\mu} \mapsto \widetilde{q_{\mu}}, \widetilde{p_{\mu}}, \mu \in \overline{1, d}$, где

$$
\left(\widetilde{q_{\mu}} \varphi\right)(x)=x_{\mu} \varphi(x), \quad\left(\widetilde{p_{\mu}} \varphi\right)(x)=\varkappa \frac{\partial \varphi}{\partial x_{\mu}}, \quad \varphi \in \mathbb{F}[x]
$$

задает представление $\Pi: \mathbb{F}\langle r\rangle \rightarrow \mathfrak{L}(\mathbb{F}[x]), f(r) \mapsto f(\widetilde{r})$, алгебры некоммутативных многочленов $\mathbb{F}\langle r\rangle$ в $\mathbb{F}[x]$.

1.7. ПРЕДЛОЖЕНИЕ. Пусть $f(q, p)$ - нормальный многочлен $u f(\widetilde{q}, \widetilde{p})$ - соответствующий оператор; $f(\widetilde{q}, \widetilde{p})=0$ тогда и только тогда, когда $f(q, p)=0$.

ДокАЗАтЕльСтво. Очевидно, что $\widetilde{p}^{j}\left(x^{k}\right)=\varkappa^{|j|} j !\left(\begin{array}{c}k \\ j\end{array}\right) x^{k-j}$ для всех $j, k \in \mathbb{Z}_{+}^{d}$. Далее, пусть $f(\widetilde{q}, \widetilde{p})=0$, где

$$
f(q, p)=\sum_{i, j} c_{i j} q^{i} p^{j}=\sum_{j} \chi_{j}(q) p^{j}, \quad \chi_{j}(q)=\sum_{i} c_{i j} q^{i} .
$$

Тогда, в частности,

$$
f(\widetilde{q}, \widetilde{p})\left(x^{k}\right)=\sum_{j \leqslant k} \chi_{j}(x) \varkappa^{|j|} j !\left(\begin{array}{c}
k \\
j
\end{array}\right) x^{k-j}=0, \quad \forall k \in \mathbb{Z}_{+}^{d} .
$$

Полагая последовательно $|k|=0,1,2, \ldots$, убедимся, что многочлены $\chi_{j}(q)=0$ при $|j|=$ $0,1,2, \ldots$ Отсюда $f(q, p)=0$, что и требовалось доказать. 
1.8. СлЕДСтвИЕ. Справедливо равенство $\operatorname{ker} \Pi=\mathcal{J}$, әде $\operatorname{ker} \Pi-$ ядро морфизма П.

ДОКАЗАТЕЛЬСТВО вытекает из предложения 1.7 с учетом следствия 1.5 и очевидного включения $\mathcal{J} \subset \operatorname{ker} \Pi$.

1.9. Теорема. Алгебра Гейзенберга $\mathcal{H}$ канонически изоморфна алгебре всех нормальных многочленов из $\mathbb{F}\langle r\rangle$ с обычными линейнбыми операчиями и умножением, задаваемым правилом

$$
q^{i} p^{j} \cdot q^{k} p^{l}=\sum_{m, n} A_{m n}^{i j, k l} q^{m} p^{n}, \quad A_{m n}^{i j, k l}=\sum_{r} \varkappa^{|r|}\left(\begin{array}{c}
j, k \\
r
\end{array}\right) \delta_{m}^{i+k-r} \delta_{n}^{j+l-r},
$$

əде $i, j, k, l, m, n, r \in \mathbb{Z}_{+}^{d}$.

ДокАЗАТЕЛЬСТво следует из предложений $1.4,1.7$ и следствий $1.5,1.8$.

Ниже мы отождествляем $\mathcal{H}$ с этой алгеброй нормальных многочленов.

1.10. Действия над нормальными многочленами. Из правила умножения теоремы 1.9 следует, что коммутатор имеет вид

$$
\begin{aligned}
{\left[q^{i} p^{j}, q^{k} p^{l}\right] } & =\varkappa \sum_{m, n} C_{m n}^{i j, k l} q^{m} p^{n}, \\
C_{m n}^{i j, k l} & =\frac{1}{\varkappa}\left(A_{m n}^{i j, k l}-A_{m n}^{k l, i j}\right)=\sum_{r} \varkappa^{|r|-1}(\underset{r}{i, j ; k, l}) \delta_{m}^{i+k-r} \delta_{n}^{j+l-r},
\end{aligned}
$$

где $i, j, k, l, m, n, r \in \mathbb{Z}_{+}^{d}$. В частности, если $f(q, p)=\sum_{i, j} f_{i j} q^{i} p^{j}$ - нормальньй многочлен, то

$$
\begin{aligned}
& {\left[p_{\mu}, f(q, p)\right]=\varkappa \frac{\partial f(q, p)}{\partial q_{\mu}},} \\
& {\left[q_{\mu}, f(q, p)\right]=-\varkappa \frac{\partial f(q, p)}{\partial p_{\mu}}, \quad \mu \in \overline{1, d},}
\end{aligned}
$$

где частные производные вычисляются по обычным формулам

$$
\begin{aligned}
& \frac{\partial f(q, p)}{\partial q_{\mu}}=\sum_{i, j} f_{i j} i_{\mu} q^{i-(\mu)} p^{j} \\
& \frac{\partial f(q, p)}{\partial p_{\mu}}=\sum_{i, j} f_{i j} j_{\mu} q^{i} p^{j-(\mu)}
\end{aligned}
$$

Формулы (1.2) имеют обобшения

$$
\left[p^{j}, f\right]=\sum_{|m| \geqslant 1} \varkappa^{|m|}\left(\begin{array}{c}
j \\
m
\end{array}\right) \frac{\partial^{|m|} f}{\partial q^{m}} p^{j-m}, \quad\left[q^{i}, f\right]=-\sum_{|m| \geqslant 1} \varkappa^{|m|}\left(\begin{array}{c}
i \\
m
\end{array}\right) q^{i-m} \frac{\partial^{|m|} f}{\partial p^{m}}
$$

где $f=f(q, p)$ - нормальный многочлен, $i, j \in \mathbb{Z}_{+}^{d}$. 
Частные производные нормальных многочленов обладают многими обычными свойствами, например, смешанные производные, взятые в различных порядках, совпадают:

$$
\frac{\partial^{2} f}{\partial q_{\mu} \partial q_{\nu}}=\frac{\partial^{2} f}{\partial q_{\nu} \partial q_{\mu}}, \quad \frac{\partial^{2} f}{\partial p_{\mu} \partial p_{\nu}}=\frac{\partial^{2} f}{\partial p_{\nu} \partial p_{\mu}}, \quad \frac{\partial^{2} f}{\partial q_{\mu} \partial p_{\nu}}=\frac{\partial^{2} f}{\partial p_{\nu} \partial q_{\mu}}, \quad \mu, \nu \in \overline{1, d}
$$

Далее, система

$$
\frac{\partial f_{\mu}}{\partial q_{\nu}}=\frac{\partial f_{\nu}}{\partial q_{\mu}}, \quad \mu, \nu \in \overline{1, d}
$$

имеет в классе нормальных многочленов обшее решение

$$
f_{\mu}(q, p)=\frac{g(q, p)}{\partial q_{\mu}}, \quad \mu \in \overline{1, d}, \quad g(q, p)=\int_{0}^{1} \sum_{\nu=1}^{d} q_{\nu} f_{\nu}(t q, p) d t+h(p)
$$

$h(p)$ - произвольный нормальный многочлен, не зависящий от $q$.

Аналогично система

$$
\frac{\partial f_{\mu}}{\partial p_{\nu}}=\frac{\partial f_{\nu}}{\partial p_{\mu}}, \quad \mu, \nu \in \overline{1, d}
$$

имеет общее решение

$$
f_{\mu}(q, p)=\frac{g(q, p)}{\partial p_{\mu}}, \quad \mu \in \overline{1, d}, \quad g(q, p)=\int_{0}^{1} \sum_{\nu=1}^{d} f_{\nu}(q, t p) p_{\nu} d t+h(q) .
$$

Наконец, система

$$
\frac{\partial^{2} f}{\partial q_{\mu} \partial p_{\nu}}=0, \quad \mu, \nu \in \overline{1, d}
$$

имеет общее решение

$$
f(q, p)=g(q)+h(p)
$$

с произвольными $g(q)$ и $h(p)$.

1.11. Свойства алгебры Гейзенберга. Перечислим основные свойства алгебры $\mathcal{H}$ с короткими пояснениями.

(1) Нормальные мономы $\rho^{i j} \equiv q^{i} p^{j}, i, j \in \mathbb{Z}_{+}^{d}$, образуют базис линейного пространства $\mathcal{H}$, моном $1=\rho^{00}$ является единицей алгебры $\mathcal{H}$.

(2) Алгебра $\mathcal{H}$ ассоциативная.

Заметим, что из ассоциативности умножения следует, что коэффициенты $A_{m n}^{i j, k l}$ (см. теорему 1.9) удовлетворяют некоторым тождествам, которые можно проверить и непосредственно.

(3) Алгебра $\mathcal{H}$ некоммутативная, ее центр $\operatorname{cen} \mathcal{H}=\mathbb{F}$. 
Действительно, пусть нормальный многочлен $f(q, p) \in \operatorname{cen} \mathcal{H}$. В частности, $\left[p_{\mu}, f(q, p)\right]=0$. Полагая $f(q, p)=\sum_{j} \chi_{j}(q) p^{j}$, из $(1.2)$ выводим

$$
\frac{\partial \chi_{j}(q)}{\partial q_{\mu}}=0
$$

при всех $\mu \in \overline{1, d}$ и $j \in \mathbb{Z}_{+}^{d}$, откуда $f=f(p)$. Далее, $\left[q_{\mu}, f(p)\right]=0$, т.е. в силу $(1.2)$

$$
\frac{\partial f(p)}{\partial q_{\mu}}=0
$$

для всех $\mu \in \overline{1, d}$, так что $f=c \cdot 1, c \in \mathbb{F}$, что и требовалось доказать.

(4) Алгебра $\mathcal{H}$ простая, т.е. не имеет нетривиальных двусторонних идеалов.

Действительно, пусть $\mathcal{I}$ - ненулевой двусторонний идеал алгебры $\mathcal{H}$ и $f \in \mathcal{I}$, $f(q, p)=\sum_{j} \chi_{j}(q) p^{j} \neq 0$. Из определения двустороннего идеала, в частности, следует, что $p_{\mu} f, f p_{\mu} \in \mathcal{I}$ для всех $\mu \in \overline{1, d}$, откуда

$$
\sum_{j} \frac{\partial \chi_{j}(q)}{\partial q_{\mu}} p^{j}=\frac{1}{\varkappa}\left[p_{\mu}, f\right] \in \mathcal{I} .
$$

По индукции

$$
\sum_{j} \frac{\partial^{k} \chi_{j}(q)}{\partial q^{k}} p^{j} \in \mathcal{I}
$$

для всех $k \in \mathbb{Z}_{+}^{d}$. Выбирая $k$ подходяшим образом, построим ненулевой многочлен $g(p)=\sum_{j} c_{j} p^{j} \in \mathcal{I}, c_{j} \in \mathbb{F}$. Далее, $q_{\mu} g, g q_{\mu} \in \mathcal{I}$ для всех $\mu \in \overline{1, d}$, откуда

$$
\frac{\partial^{l} g(p)}{\partial p^{l}} \in \mathcal{I}
$$

при всех $l \in \mathbb{Z}_{+}^{d}$. Выбирая $l$ подходящим образом, получим ненулевую постоянную $c=$ $c \cdot 1 \in \mathcal{I}, 0 \neq c \in \mathbb{F}$. Итак, $1 \in \mathcal{I}$ и, значит, $\mathcal{I}=\mathcal{H}$, что и требовалось доказать.

(5) Алгебра мультипликаторов $\mathfrak{M}(\mathcal{H})=\mathbb{F}$.

Утверждение (5) следует из равенства $\mathfrak{M}(\mathcal{H})=\operatorname{cen} \mathcal{H}($ см. пункт (8) раздела 0.2$)$ и свойства (3).

(6) Алгебра $\mathcal{H}$ имеет $\mathbb{Z}^{d}$-градуировку.

Действительно, каждой паре мультииндексов $i, j \in \mathbb{Z}^{d}$ поставим в соответствие градуировку $\gamma(i, j)=i-j$, а для каждого мультииндекса $k \in \mathbb{Z}^{d}$ рассмотрим

$$
\mathcal{H}_{k}=\left\{f(q, p)=\sum_{i-j=k} f_{i j} \rho^{i j} \in \mathcal{H}\right\}
$$

- линейное пространство $\gamma$-однородных нормальных многочленов порядка $k$. Тогда

$$
\mathcal{H}=\sum_{k} \mathcal{H}_{k}, \quad \mathcal{H}_{k} \cdot \mathcal{H}_{l} \subset \mathcal{H}_{k+l},
$$

т.е. $f g \in \mathcal{H}_{k+l}$, если $f \in \mathcal{H}_{k}, g \in \mathcal{H}_{l}$ (см. правило умножения теоремы 1.9).

(7) В алгебре $\mathcal{H}$ определены две операции транспонирования. 
Первая операция транспонирования определяется как линейное отображение ${ }^{t}: \mathcal{H} \rightarrow$ $\mathcal{H}$, однозначно задаваемое правилом $\left(q^{i} p^{j}\right)^{t}=q^{j} p^{i}, \quad i, j \in \mathbb{Z}_{+}^{d}$. Действительно, это правило согласовано с определяюшими соотношениями алгебры Гейзенберга (см. раздел 1.2), например, $\left(p_{\mu} q_{\nu}-q_{\nu} p_{\mu}-\varkappa \delta_{\mu \nu}\right)^{t}=p_{\nu} q_{\mu}-q_{\mu} p_{\nu}-\varkappa \delta_{\nu \mu}, \mu, \nu \in \overline{1, d}$, и, кроме того, $\left(f^{t}\right)^{t}=f,(f g)^{t}=g^{t} f^{t}, f, g \in \mathcal{H}$. Обратим внимание на то, что ${ }^{t}: \mathcal{H}_{k} \rightarrow \mathcal{H}_{-k}$, $k \in \mathbb{Z}^{d}$, т.е. транспонирование ${ }^{t}$ меняет градуировку на противоположную, оставляя неподвижным нейтральное пространство $\mathcal{H}_{0}$.

Аналогичным образом линейное отображение ${ }^{\tau}: \mathcal{H} \rightarrow \mathcal{H}$, задаваемое правилом

$$
\left(q^{i} p^{j}\right)^{\tau}=(-p)^{j} q^{i}=(-1)^{|j|} \sum_{k} \varkappa^{|k|}\left(\begin{array}{c}
i, j \\
k
\end{array}\right) q^{i-k} p^{j-k}, \quad i, j \in \mathbb{Z}_{+}^{d},
$$

определяет альтернативную операцию транспонирования в $\mathcal{H}$. В этом случае ${ }^{\tau}: \mathcal{H}_{k} \rightarrow$ $\mathcal{H}_{k}, k \in \mathbb{Z}^{d}$, т.е. транспонирование ${ }^{\tau}$ сохраняет градуировку.

Правило

$$
(A f)(q, p)=f\left(\frac{p+q}{2}, p-q\right), \quad f(q, p) \in \mathcal{H}
$$

определяет линейное отображение $A: \mathcal{H} \rightarrow \mathcal{H}$. Оно обратимо,

$$
\left(A^{-1} g\right)(q, p)=g\left(q-\frac{1}{2} p, q+\frac{1}{2} p\right), \quad g \in \mathcal{H}
$$

согласовано с определяюшими соотношениями раздела 1.2, например

$$
\begin{aligned}
A\left(p_{\mu} q_{\nu}-q_{\nu} p_{\mu}-\varkappa \delta_{\mu \nu}\right)= & \frac{1}{2}\left\{\left(p_{\mu} p_{\nu}-p_{\nu} p_{\mu}\right)+\left(p_{\nu} q_{\mu}-q_{\mu} p_{\nu}-\varkappa \delta_{\nu \mu}\right)+\right. \\
& \left.+\left(p_{\mu} q_{\nu}-q_{\nu} p_{\mu}-\varkappa \delta_{\mu \nu}\right)+\left(q_{\nu} q_{\mu}-q_{\mu} q_{\nu}\right)\right\}, \quad \mu, \nu \in \overline{1, d}
\end{aligned}
$$

и, следовательно, является автоморфизмом алгебры $\mathcal{H}$.

Легко проверяется, что автоморфизм А переводит операции транспонирования ${ }^{\tau}{ }^{t}$ друг в друга, т.е. $(A(f))^{t}=A\left(f^{\tau}\right), f \in \mathcal{H}$.

Транспонирования в $\mathcal{H}$, в свою очередь, порождают транспонирования в алгебре $\mathfrak{L}_{0}(\mathcal{H})$ правилами

$$
F^{t}(f)=\left(F\left(f^{t}\right)\right)^{t}, \quad F^{\tau}(f)=\left(F\left(f^{\tau}\right)\right)^{\tau}, \quad F \in \mathfrak{L}(\mathcal{H}), \quad f \in \mathcal{H} .
$$

(8) В алгебре $\mathcal{H}$ определено понятие четности.

Действительно, $\mathcal{H}=\mathcal{H}_{\mathrm{ev}}+\mathcal{H}_{\mathrm{od}}$, где

$$
\mathcal{H}_{\mathrm{ev}}=\{f \in \mathcal{H}: f(-q,-p)=f(q, p)\}, \quad \mathcal{H}_{\mathrm{od}}=\{f \in \mathcal{H}: f(-q,-p)=-f(q, p)\},
$$

причем

$$
\mathcal{H}_{\mathrm{ev}} \cdot \mathcal{H}_{\mathrm{ev}}, \mathcal{H}_{\mathrm{od}} \cdot \mathcal{H}_{\mathrm{od}} \subset \mathcal{H}_{\mathrm{ev}}, \quad \mathcal{H}_{\mathrm{ev}} \cdot \mathcal{H}_{\mathrm{od}}, \mathcal{H}_{\mathrm{od}} \cdot \mathcal{H}_{\mathrm{ev}} \subset \mathcal{H}_{\mathrm{od}}
$$

(9) В алгебре $\mathcal{H}$ определена скобка Пуассона 
Действительно, билинейная операция

$$
\mathcal{H} \ni f, g \mapsto\{f, g\}=\frac{1}{\varkappa}[f, g] \in \mathcal{H}
$$

обладает всеми необходимыми свойствами. В частности,

$$
\left\{\rho^{i j}, \rho^{k l}\right\}=\sum_{m, n} C_{m n}^{i j, k l} \rho^{m n}, \quad i, j, k, l \in \mathbb{Z}_{+}^{d},
$$

структурные константы $C_{m n}^{i j, k l}$ определены в разделе 1.10 .

Таким образом, в множестве нормальных многочленов $\mathcal{H}$ определены следуюшие структуры: линейное пространство - множество $\mathcal{H}$ плюс обычные $\mathbb{F}$-линейные операции, (ассочиативная) алгебра - линейное пространство $\mathcal{H}$ плюс ассоциативное некоммутативное умножение, алгебра Пуассона - алгебра $\mathcal{H}$ плюс скобка Пуассона, алгебра Ли - линейное пространство $\mathcal{H}$ плюс скобка Пуассона, рассматриваемая как скобка Ли.

\section{2. АЛГЕБРА ЛИ ДИФФЕРЕНЦИРОВАНИЙ АЛГЕБРЫ ГЕЙЗЕНБЕРГА}

2.1. Внутренние дифференцирования. Согласно пункту (9) раздела 0.3 в алгебре $\mathcal{H}$ есть внутренние дифференцирования $\alpha_{-}(f)$, задаваемые правилом

$$
\alpha_{-}(f)(g)=\frac{1}{\varkappa}[f, g]=\{f, g\}, \quad f, g \in \mathcal{H}
$$

(множитель $1 / \varkappa$ добавлен для удобства, ср. с (1.2)); очевидно, что дифференцирование $\alpha_{-}(f)$ нетривиальное, если нормальный многочлен $f(q, p) \neq \mathbb{F} \cdot 1$. Итак,

(1) правило $f \mapsto \alpha_{-}(f)$ задает линейное отображение $\alpha_{-}: \mathcal{H} \rightarrow \mathfrak{D}(\mathcal{H})$, его ядро $\operatorname{ker} \alpha_{-}=\operatorname{cen} \mathcal{H}=\mathbb{F}$.

2.2. ПРЕДЛОЖЕНИЕ. Все дифферениирования алгебры $\mathcal{H}$ внутренние, т.е. отображсение $\alpha_{-}$сюрвективное.

ДокаЗАТЕльство. Пусть $D \in \mathfrak{D}(\mathcal{H})$. Очевидно, что $D$ полностью определяется своим действием на образуюшие $q_{\mu}, p_{\mu}$, т.е. нормальными многочленами

$$
f_{\mu}(q, p)=D\left(q_{\mu}\right), \quad g_{\mu}(q, p)=D\left(p_{\mu}\right), \quad \mu \in \overline{1, d} .
$$

В свою очередь, нормальные многочлены $f_{\mu}, g_{\mu} \in \mathcal{H}, \mu \in \overline{1, d}$, будут задавать дифференцирование $D \in \mathfrak{D}(\mathcal{H})$, если они согласуются с коммутационными соотношениями раздела 1.2 , т.е.

$$
\begin{aligned}
D\left(q_{\mu} q_{\nu}-q_{\nu} q_{\mu}\right) & =\left(f_{\mu} q_{\nu}+q_{\mu} f_{\nu}\right)-\left(f_{\nu} q_{\mu}+q_{\nu} f_{\mu}\right)=\left[f_{\mu}, q_{\nu}\right]-\left[f_{\nu}, q_{\mu}\right]= \\
& =\varkappa\left(\frac{\partial f_{\mu}}{\partial p_{\nu}}-\frac{\partial f_{\nu}}{\partial p_{\mu}}\right)=0 \\
D\left(p_{\mu} p_{\nu}-p_{\nu} p_{\mu}\right) & =\left(g_{\mu} p_{\nu}+p_{\mu} g_{\nu}\right)-\left(g_{\nu} p_{\mu}+p_{\nu} g_{\mu}\right)=\left[g_{\mu}, p_{\nu}\right]-\left[g_{\nu}, p_{\mu}\right]= \\
& =\varkappa\left(\frac{\partial g_{\nu}}{\partial q_{\mu}}-\frac{\partial g_{\mu}}{\partial q_{\nu}}\right)=0 \\
D\left(p_{\mu} q_{\nu}-q_{\nu} p_{\mu}-\varkappa \delta_{\mu \nu}\right) & =\left(g_{\mu} q_{\nu}+p_{\mu} f_{\nu}\right)-\left(f_{\nu} p_{\mu}+q_{\nu} g_{\mu}\right)-0=\left[g_{\mu}, q_{\nu}\right]+\left[p_{\mu}, f_{\nu}\right]= \\
& =\varkappa\left(\frac{\partial g_{\mu}}{\partial p_{\nu}}+\frac{\partial f_{\nu}}{\partial q_{\mu}}\right)=0
\end{aligned}
$$


для всех $\mu, \nu \in \overline{1, d}$, причем мы воспользовались равенствами (1.2). Согласно формулам (1.6)-(1.7) и (1.4)-(1.6) система (2.1) дает

$$
f_{\mu}=\frac{\partial f}{\partial p_{\mu}}
$$

а система $(2.2)-$

$$
g_{\mu}=\frac{\partial g}{\partial q_{\mu}}, \quad \mu \in \overline{1, d}
$$

причем в силу (2.3) нормальные многочлены $f(q, p)$ и $g(q, p)$ удовлетворяют системе

$$
\frac{\partial^{2}(f+g)}{\partial q_{\mu} \partial p_{\nu}}=0, \quad \mu, \nu \in \overline{1, d}
$$

В соответствии с формулами (1.8), (1.9) $f(q, p)+g(q, p)=\varphi(q)+\psi(p)$, откуда $f(q, p)-$ $\varphi(q)=-g(q, p)+\psi(p)=h(q, p), h \in \mathcal{H}$, или $f(q, p)=h(q, p)+\varphi(q), g(q, p)=-h(q, p)+$ $\psi(p)$. Итак,

$$
D\left(q_{\mu}\right)=f_{\mu}=\frac{\partial f}{\partial p_{\mu}}=\frac{\partial h}{\partial p_{\mu}}=\frac{1}{\varkappa}\left[h, q_{\mu}\right], \quad D\left(p_{\mu}\right)=g_{\mu}=\frac{\partial g}{\partial q_{\mu}}=-\frac{\partial h}{\partial q_{\mu}}=\frac{1}{\varkappa}\left[h, p_{\mu}\right]
$$

для всех $\mu \in \overline{1, d}$, так что $D=\{h, \cdot\}$, что и требовалось доказать.

2.3. Алгебра Ли $\mathcal{H}_{\star}$. Разложим линейное пространство нормальных многочленов $\mathcal{H}$ в прямую сумму:

$$
\mathcal{H}=\mathbb{F} \cdot 1+\mathcal{H}_{\star}, \quad \mathcal{H}_{\star}=\{f(q, p) \in \mathcal{H}: f(0,0)=0\}
$$

и пусть $\operatorname{pr}_{1}: \mathcal{H} \rightarrow \mathbb{F} \cdot 1, \operatorname{pr}_{\star}: \mathcal{H} \rightarrow \mathcal{H}_{\star}-$ соответствуюшие проекторы,

$$
\operatorname{pr}_{1}(f)=f(0,0), \quad \operatorname{pr}_{\star}(f)(q, p)=f(q, p)-f(0,0), \quad f(q, p) \in \mathcal{H} .
$$

Определим на $\mathcal{H}_{\star}$ скобку $[\cdot, \cdot]_{\star}: \mathcal{H}_{\star} \times \mathcal{H}_{\star} \rightarrow \mathcal{H}_{\star}$ правилом

$$
[f, g]_{\star}=\operatorname{pr}_{\star}(\{f, g\}), \quad f, g \in \mathcal{H}_{\star} .
$$

С помощью (1.1) легко проверяется, что

(1) скобка $[\cdot, \cdot]_{\star}$ обладает всеми свойствами скобки Ли и, следовательно, определяет на $\mathcal{H}_{\star}$ структуру алгебры Ли;

(2) мономы $\rho^{i j} \equiv q^{i} p^{j}, i, j \in \mathbb{Z}_{+}^{d},|i+j|>0$, образуют базис в $\mathcal{H}_{\star}$;

(3) скобка $[\cdot, \cdot]_{\star}$ определяется равенствами

$$
\left[\rho^{i j}, \rho^{k l}\right]_{\star}=\sum_{|m+n|>0} C_{m n}^{i j, k l} \rho^{m n}, \quad i, j, k, l \in \mathbb{Z}_{+}^{d}, \quad|i+j|>0, \quad|k+l|>0,
$$

структурные константы $C_{m n}^{i j, k l}$ определены в разделе 1.10. 
2.4. ТЕОремА. Определен изоморфизм алгебр Ли

$$
\alpha_{-}: \mathcal{H}_{\star} \simeq \mathfrak{D}(\mathcal{H})
$$

где $\alpha_{-}(f)=\{f, \cdot\} \equiv F, \quad f \in \mathcal{H}_{\star}$, причем

$$
\begin{aligned}
\alpha_{-}\left([f, g]_{\star}\right) & =\left[\alpha_{-}(f), \alpha_{-}(g)\right], \quad f, g \in \mathcal{H}_{\star} ; \\
\alpha_{-}(f)^{t} & =-\alpha_{-}\left(f^{t}\right), \quad f \in \mathcal{H}_{\star} .
\end{aligned}
$$

ДокАЗАТЕЛЬСтво. В силу предложения 2.2 и приведенных выше рассуждений доказательство сводится к простой проверке (транспонирование определено в пукте (7) раздела 1.11).

Итак, алгебра Ли

$$
\mathfrak{D}(\mathcal{H})=\left\{F=\{f, \cdot\}: f=\sum_{|i+j|>0} f_{i j} \rho^{i j} \in \mathcal{H}\right\}
$$

имеет базис $R^{i j}=\left\{\rho^{i j}, \cdot\right\}, i, j \in \mathbb{Z}_{+}^{d},|i+j|>0$, скобка и действие даются формулами

$$
\left[R^{i j}, R^{k l}\right]=\sum_{|m+n|>0} C_{m n}^{i j, k l} R^{m n}, \quad R^{i j}\left(\rho^{k l}\right)=\sum_{|m+n|>0} C_{m n}^{i j, k l} \rho^{m n},
$$

структурные константы определены в разделе 1.10 .

В частности, $\left[R^{0(\mu)}, R^{i+(\mu), j}\right]=\left(i_{\mu}+1\right) R^{i j}$ для любых $\mu \in \overline{1, d}, i, j \in \mathbb{Z}_{+}^{d},|i+j|>0$, откуда следует, что

(1) коммутант $[\mathfrak{D}(\mathcal{H}), \mathfrak{D}(\mathcal{H})]=\mathfrak{D}(\mathcal{H})$.

2.5. Степень дифференцирования и градуировка. Обозначим через

$$
\mathcal{H}^{u}=\left\{f=\sum_{|i+j|=u} f_{i j} \rho^{i j} \in \mathcal{H}\right\}, \quad u \in \mathbb{Z}_{+},
$$

линейное пространство всех однородных нормальных многочленов степени $u$. Тогда

$$
\mathcal{H}_{\star}=\sum_{u=1}^{\infty} \mathcal{H}^{u} \text { и }\left[\mathcal{H}^{v}, \mathcal{H}^{w}\right]_{\star} \subset \sum_{u=1}^{v+w-2} \mathcal{H}^{n}, \quad v, w \in \mathbb{N} .
$$

Пусть $\mathfrak{D}^{u}(\mathcal{H})=\left\{F=\{f, \cdot\}: f \in \mathcal{H}^{u}\right\}-$ линейное пространство однородных дифферениирований степени $u \in \mathbb{N}$. Согласно теореме 2.4 алгебра Ли $\mathfrak{D}(\mathcal{H})$ разлагается в прямую сумму линейных пространств

$$
\mathfrak{D}(\mathcal{H})=\sum_{u=1}^{\infty} \mathfrak{D}^{u}(\mathcal{H}) \quad \text { и } \quad\left[\mathfrak{D}^{v}(\mathcal{H}), \mathfrak{D}^{w}(\mathcal{H})\right] \subset \sum_{u=1}^{v+w-2} \mathfrak{D}^{u}(\mathcal{H}), \quad v, w \in \mathbb{N} .
$$

Аналогичным образом положим $\mathcal{H}_{\star k}=\mathcal{H}_{\star} \cap \mathcal{H}_{k}, k \in \mathbb{Z}^{d}$ (см. пункт (6) раздела 1.11 , заметим, что $\mathcal{H}_{\star k}=\mathcal{H}_{k}$ при $\left.k \neq 0\right)$, и пусть $\mathfrak{D}_{k}(\mathcal{H})=\left\{F=\{f, \cdot\}: f \in \mathcal{H}_{\star k}\right\}$ - линейные пространства $\gamma$-однородных дифференцирований порядка $k \in \mathbb{Z}^{d}$. Легко проверяется, что алгебра Ли $\mathfrak{D}(\mathcal{H})$ обладает градуировкой:

$$
\mathfrak{D}(\mathcal{H})=\sum_{k \in \mathbb{Z}^{d}} \mathfrak{D}_{k}(\mathcal{H}), \quad\left[\mathfrak{D}_{k}(\mathcal{H}), \mathfrak{D}_{l}(\mathcal{H})\right] \subset \mathfrak{D}_{k+l}(\mathcal{H}), \quad k, l \in \mathbb{Z}^{d}
$$


2.6. Классический предел. Согласно разделу 2.4 алгебра Ли $\mathfrak{D}(\mathcal{H})$ имеет базис $\zeta^{i j}=R^{i j}, i, j \in \mathbb{Z}_{+}^{d},|i+j|>0$, и коммутатор

$$
\left[\zeta^{i j}, \zeta^{k l}\right]=\sum_{m, n} C_{m n}^{i j, k l} \zeta^{m n}
$$

где структурные константы

$$
\begin{aligned}
C_{m n}^{i j, k l} & =C_{m n}^{i j, k l}(\varkappa)=\sum_{|r|>0} \varkappa^{|r|-1}(\underset{r}{i, j ; k, l}) \delta_{m}^{i+k-r} \delta_{n}^{j+l-r}= \\
& =\sum_{\mu=1}^{d}\left(j_{\mu} k_{\mu}-l_{\mu} i_{\mu}\right) \delta_{m}^{i+k-(\mu)} \delta_{n}^{j+l-(\mu)}+O(\varkappa) .
\end{aligned}
$$

Переходя в последнем равенстве к пределу $\varkappa \rightarrow 0$, получим алгебру Ли $\mathcal{Z}$ с базисом $\zeta^{i j}$, $i, j \in \mathbb{Z}_{+}^{d},|i+j|>0$, и скобкой

$$
\left[\zeta^{i j}, \zeta^{k l}\right]=\sum_{|m+n|>0}^{d} Z_{m n}^{i j, k l} \zeta^{m n}
$$

где структурные константы

$$
Z_{m n}^{i j, k l}=\sum_{\mu=1}^{d}\left(j_{\mu} k_{\mu}-l_{\mu} i_{\mu}\right) \delta_{m}^{i+k-(\mu)} \delta_{n}^{j+l-(\mu)} .
$$

Заметим, что алгебра $\mathcal{H}$ в пределе $\varkappa \rightarrow 0$ переходит в алгебру $\mathbb{F}[r]$ коммутативных многочленов с образуюшими $r=\left(q_{1}, \ldots, p_{d}\right)$ и обычными линейными операциями и умножением. Базисные скобки Пуассона (см. пункт (9) раздела 1.11 ) принимают в этом случае вид

$$
\begin{aligned}
\left\{\rho^{i j}, \rho^{k l}\right\} & =\sum_{m, n} Z_{m n}^{i j, k l} \rho^{m n}=\sum_{\mu=1}^{d}\left(j_{\mu} k_{\mu}-l_{\mu} i_{\mu}\right) \rho^{i+k-(\mu), j+l-(\mu)}= \\
& =\sum_{\mu=1}^{d}\left(\frac{\partial \rho^{i j}}{\partial p_{\mu}} \frac{\partial \rho^{k l}}{\partial q_{\mu}}-\frac{\partial \rho^{i j}}{\partial q_{\mu}} \frac{\partial \rho^{k l}}{\partial p_{\mu}}\right), \quad i, j, k, l \in \mathbb{Z}_{+}^{d},
\end{aligned}
$$

откуда

$$
\{f, g\}=\sum_{\mu=1}^{d}\left(\frac{\partial f}{\partial p_{\mu}} \frac{\partial g}{\partial q_{\mu}}-\frac{\partial f}{\partial q_{\mu}} \frac{\partial g}{\partial p_{\mu}}\right), \quad f, g \in \mathbb{F}[r],
$$

в полном согласии с классическими выражениями (см., например, [2]). Другими словами,

(1) в пределе $\varkappa \rightarrow 0$ алгебра Пуассона $\mathcal{H}$ переходит в классическую алгебру Пуассона $\mathbb{F}[r]$ коммутативных многочленов со скобкой (2.4).

С другой стороны, рассматривая $\mathbb{F}[r]$ как алгебру Ли со скобкой $(2.4)$, приходим к утверж дению:

(2) определен изоморфизм алгебр Ли $\mathcal{Z} \simeq \mathbb{F}[r] /\{1\}$, где $\{1\}-$ центр алгебры Ли $\mathbb{F}[r]$. 


\section{3. ОСНОВНЫЕ ПОДАЛГЕБРЫ}

3.1. Дифференцирования первой степени. Дифференцирования $Q_{\mu}=\left\{q_{\mu}, \cdot\right\}$, $P_{\mu}=\left\{p_{\mu}, \cdot\right\}, \quad \mu \in \overline{1, d}$, образуют базис линейного пространства $\mathfrak{D}^{1}(\mathcal{H})$ диффференцирований первой степени. С помошью формул (1.1), (1.2) легко проверяются следуюшие утверж дения:

(1) дифференцирования $Q_{\mu}, P_{\mu}$ действуют на $\mathcal{H}$ по правилам

$$
Q_{\mu}(f)=-\frac{\partial f}{\partial p_{\mu}}, \quad P_{\mu}(f)=\frac{\partial f}{\partial q_{\mu}}, \quad f \in \mathcal{H}, \quad \mu \in \overline{1, d}
$$

(2) дифференцирования $Q_{\mu}, P_{\mu}, \mu, \nu \in \overline{1, d}$, попарно коммутируют, так что $\mathfrak{D}^{1}(\mathcal{H})$ - коммутативная подалгебра алгебры Ли $\mathfrak{D}(\mathcal{H})$;

(3) семейство $Q_{1}, \ldots, P_{d}$ тотальное, т.е. нормальный многочлен $f \in \mathcal{H}$ удовлетворяет системе $Q_{\mu}(f)=P_{\mu}(f)=0, \mu \in \overline{1, d}$, тогда и только тогда, когда $f \in \mathcal{H}^{0}=\mathbb{F}$;

(4) определена стандартная дифференциальная алгебра $\left(\mathcal{H}, \mathfrak{D}^{1}(\mathcal{H})\right)($ см. раздел 0.5).

3.2. Экспоненты. Пусть $c=(a, b)=\left(a_{1}, \ldots, b_{d}\right) \in \mathbb{F}^{2 d}$. Будем называть әкспонентами (точнее, $c$-экспонентами, см., например, [16]) решения $f$ системы

$$
Q_{\mu}(f)=a_{\mu} f, \quad P_{\mu}(f)=b_{\mu} f, \quad \mu \in \overline{1, d} .
$$

Поскольку операторы $Q_{\mu}, P_{\mu}$ суть дифференцирования, экспоненты обладают характеризуюшим свойством:

(1) произведение $f^{\prime} \cdot f^{\prime \prime}$ есть $\left(c^{\prime}+c^{\prime \prime}\right)$-экспонента, если $f^{\prime}$ есть $c^{\prime}$-экспонента, а $f^{\prime \prime}$ есть $c^{\prime \prime}$-экспонента.

Простые вычисления показывают, что

(2) всякое решение системы (3.1) в классе формальных степенных рядов имеет вид (с точностью до нормировки)

$$
f(q, p ; c)=\sum_{i \in \mathbb{Z}_{+}^{d}} \frac{b^{i}}{i !} q^{i} \sum_{j \in \mathbb{Z}_{+}^{d}}(-1)^{|j|} \frac{a^{j}}{j !} p^{j}=\sum_{i, j \in \mathbb{Z}_{+}^{d}}(-1)^{|j|} \frac{b^{i} a^{j}}{i ! j !} q^{i} p^{j} .
$$

В частности, экспоненты являются формальными (обобшенными) элементами, не принадлежашими алгебре $\mathcal{H}$. Тем не менее они могут оказаться полезными в приложениях.

3.3. Дифференцирования второй степени. Рассмотрим дифференцирования $Q_{\mu \nu}=\left\{q_{\mu} q_{\nu}, \cdot\right\}=Q_{\nu \mu}, \quad R_{\mu \nu}=\left\{q_{\mu} p_{\nu}, \cdot\right\}, \quad P_{\mu \nu}=\left\{p_{\mu} p_{\nu}, \cdot\right\}=P_{\nu \mu}, \mu, \nu \in \overline{1, d}$. С помошью формул (1.1) легко проверить, что

(1) введенные дифференцирования действуют по формулам

$$
\begin{aligned}
Q_{\mu \nu}(f) & =-\left(q_{\mu} \frac{\partial f}{\partial p_{\nu}}+q_{\nu} \frac{\partial f}{\partial p_{\mu}}\right)-\varkappa \frac{\partial^{2} f}{\partial p_{\mu} \partial p_{\nu}}, \\
R_{\mu \nu}(f) & =q_{\mu} \frac{\partial f}{\partial q_{\nu}}-\frac{\partial f}{\partial p_{\mu}} p_{\nu}, \\
P_{\mu \nu}(f) & =\left(\frac{\partial f}{\partial q_{\mu}} p_{\nu}+\frac{\partial f}{\partial q_{\nu}} p_{\mu}\right)+\varkappa \frac{\partial^{2} f}{\partial q_{\mu} \partial q_{\nu}}
\end{aligned}
$$


для всех $f \in \mathcal{H}, \mu, \nu \in \overline{1, d}$ (обратим внимание на наличие вторых производных);

(2) $\mathfrak{D}^{2}(\mathcal{H})$ - подалгебра алгебры Ли $\mathfrak{D}(\mathcal{H})$ размерности $d(2 d+1)$ с базисом $Q_{\mu \nu}$, $\mu \leqslant \nu, R_{\mu \nu}, \mu, \nu \in \overline{1, d}, P_{\mu \nu}, \mu \leqslant \nu$, и коммутационными соотношениями

$$
\begin{aligned}
& {\left[Q_{\lambda \mu}, Q_{\nu \pi}\right]=0, \quad\left[P_{\lambda \mu}, P_{\nu \pi}\right]=0, \quad\left[R_{\lambda \mu}, Q_{\nu \pi}\right]=\delta_{\mu \nu} Q_{\lambda \pi}+\delta_{\mu \pi} Q_{\lambda \nu},} \\
& {\left[R_{\lambda \mu}, R_{\nu \pi}\right]=\delta_{\mu \nu} R_{\lambda \pi}-\delta_{\lambda \pi} R_{\nu \mu}, \quad\left[R_{\lambda \mu}, P_{\nu \pi}\right]=-\delta_{\lambda \nu} P_{\mu \pi}-\delta_{\lambda \pi} P_{\mu \nu},} \\
& {\left[Q_{\lambda \mu}, P_{\nu \pi}\right]=-\delta_{\lambda \nu} R_{\mu \pi}-\delta_{\lambda \pi} R_{\mu \nu}-\delta_{\mu \nu} R_{\lambda \pi}-\delta_{\mu \pi} R_{\lambda \nu}, \quad \lambda, \mu, \nu, \pi \in \overline{1, d}}
\end{aligned}
$$

Рассмотрим алгебру Ли $\mathfrak{s p}(2 d ; \mathbb{F}$ ) (т.е. алгебру Ли формальной симплектической групाы порядка $2 d)$ в следуюшей реализации: алгебра $\mathfrak{s p}(2 d ; \mathbb{F})$ состоит из всех матриц $M, N, \ldots$ порядка $2 d$ с элементами из $\mathbb{F}$, записанных в блочном виде

$$
M=\left(\begin{array}{cc}
b & a \\
c & -b^{t}
\end{array}\right), \quad N=\left(\begin{array}{cc}
y & x \\
z & -y^{t}
\end{array}\right), \quad \cdots
$$

$\left(a, b, c, x, y, z, \ldots-\right.$ квадратные матришы порядкка $d \times d$ с элементами из $\mathbb{F}$, причем $a=a^{t}$, $c=c^{t}, x=x^{t}, z=z^{t}, \ldots$ ), и со скобкой Ли $[M, N]=M N-N M$ (см., например, [22]).

С помошью коммуташионных соотношений пункта (2) раздела 3.3 можно проверить, что

(3) правило

$$
M=\left(\begin{array}{cc}
b & a \\
c & -b^{t}
\end{array}\right) \mapsto D=-a \frac{Q}{2}+b R+c \frac{P}{2}
$$

задает изоморфизм алгебр Ли $\mathfrak{s p}(2 d ; \mathbb{F}) \simeq \mathfrak{D}^{2}(\mathcal{H})$.

Подробнее,

$$
D=\sum_{\lambda, \mu=1}^{d}\left(-\frac{1}{2} a^{\lambda \mu} Q_{\lambda \mu}+b^{\lambda \mu} R_{\lambda \mu}+\frac{1}{2} c^{\lambda \mu} P_{\lambda \mu}\right)
$$

3.4. Симметрии дифференциальной алгебры $\left(\mathcal{H}, \mathfrak{D}^{1}(\mathcal{H})\right)$. По определению (см. раздел 0.5) дифференцирование $F \in \mathfrak{D}(\mathcal{H})$ называется дифференцированием ЛиБеклунда дифференциальной алгебры $\left(\mathcal{H}, \mathfrak{D}^{1}(\mathcal{H})\right)$, если коммутаторы $\left[F, Q_{\mu}\right],\left[F, P_{\mu}\right] \in$ $\mathfrak{D}^{1}(\mathcal{H})$ для всех $\mu \in \overline{1, d}$. С помошью утверждения (1) раздела 3.1 легко проверяется, что это имеет место тогда и только тогда, когда $F \in \mathfrak{D}^{1}(\mathcal{H})+\mathfrak{D}^{2}(\mathcal{H})$. В силу утверждений (2) раздела 3.1 и (2) раздела 3.3 отсюда следует, что

(1) алгебра Ли симметрий дифференциальной алгебры $\left(\mathcal{H}, \mathfrak{D}^{1}(\mathcal{H})\right)$ имеет вид

$$
\operatorname{Sym}_{\mathfrak{D}^{1}(\mathcal{H})} \mathcal{H}=\mathfrak{D}^{2}(\mathcal{H})
$$


3.5. Подалгебра $\mathfrak{R}$ алгебры Ли $\mathfrak{D}^{2}(\mathcal{H})$. Из коммутационных соотношений $(2)$ раздела 3.3 следует, что

(1) линейное пространство

$$
\mathfrak{R}=\left\{A=\sum_{\mu, \nu=1}^{d} a^{\mu \nu} R_{\mu \nu}: a^{\mu \nu} \in \mathbb{F}\right\}
$$

является подалгеброй алгебры Ли $\mathfrak{D}^{2}(\mathcal{H})$.

Более того, легко заметить, что

(2) имеет место изоморфизм алгебр Ли $\mathfrak{g l}(d ; \mathbb{F}) \simeq \mathfrak{R}$, задаваемый правилом $a=$ $\left\|a^{\mu \nu}\right\| \mapsto A=\sum a^{\mu \nu} R_{\mu \nu}$.

В частности (см., например, [23]),

(3) форма Киллинга на $\mathfrak{R}$ имеет вид

$$
\phi(A, B)=2 d \operatorname{tr}(a b)-2 \operatorname{tr}(a) \operatorname{tr}(b), \quad A=\sum a^{\mu \nu} R_{\mu \nu}, \quad B=\sum b^{\mu \nu} R_{\mu \nu} ;
$$

(4) а операторы Казимира -

$$
K_{1}=\sum_{\mu_{1}=1}^{d} R_{\mu_{1} \mu_{1}}, \ldots, \quad K_{d}=\sum_{\mu_{1}, \ldots, \mu_{d}=1}^{d} R_{\mu_{1} \mu_{2}} \circ R_{\mu_{2} \mu_{3}} \circ \cdots \circ R_{\mu_{d} \mu_{1}} .
$$

С помощью коммутационных соотношений (1.1) легко проверяется, что

$$
R_{\mu \nu}: \mathcal{H}_{k} \rightarrow \mathcal{H}_{k+(\mu)-(\nu)}, \quad \mu, \nu \in \overline{1, d}, \quad k \in \mathbb{Z}^{d} .
$$

Очевидно, что $|k+(\mu)-(\nu)|=|k|$. Это замечание приводит к следующему утверждению. Для каждого $u \in \mathbb{Z}$ положим $\mathcal{H}_{u}=\sum_{|k|=u} \mathcal{H}_{k}$. Тогда $A: \mathcal{H}_{u} \rightarrow \mathcal{H}_{u}$ для всех $A \in \mathfrak{R}$ и $u \in \mathbb{Z}$. Следовательно,

(5) определены точные представления $\mathfrak{g l}(d ; \mathbb{F}) \rightarrow \mathfrak{L}\left(\mathcal{H}_{u}\right)$ алгебры Ли $\mathfrak{g l}(d ; \mathbb{F})$ в линейных пространствах $\mathcal{H}_{u}, u \in \mathbb{Z}$.

3.6. Градуирующие дифференцирования. Положим $\Gamma_{\mu}=R_{\mu \mu}$ (без суммирования), $\mu \in \overline{1, d}$, и $\Gamma=\left(\Gamma_{1}, \ldots, \Gamma_{d}\right)$. Легко проверяется, что для данных нормального многочлена $f \in \mathcal{H}$ и вектора $c=\left(c_{1}, \ldots, c_{d}\right) \in \mathbb{F}^{d}$ имеют место равенства $\Gamma_{\mu}(f)=c_{\mu} f$, $\mu \in \overline{1, d}$ (коротко, $\Gamma(f)=c f$ ), тогда и только тогда, когда $c=k=\left(k_{1}, \ldots, k_{d}\right) \in \mathbb{Z}^{d}$ и $f \in \mathcal{H}_{k}$. Другими словами,

(1) векторный оператор Г является градуируюшим на $\mathcal{H}$.

3.7. Дифференцирования с нулевой градуировкой. Обратимся к линейному пространству $\mathfrak{D}_{0}(\mathcal{H})$. Согласно разделу $2.5\left[\mathfrak{D}_{0}(\mathcal{H}), \mathfrak{D}_{k}(\mathcal{H})\right] \subset \mathfrak{D}_{k}(\mathcal{H})$ для любого $k \in \mathbb{Z}^{d}$. Более того, с помошью формулы (1.1) легко устанавливается, что

(1) $\mathfrak{D}_{0}(\mathcal{H})$ - коммутативная подалгебра алгебры Ли $\mathfrak{D}(\mathcal{H})$;

(2) в каждом из линейньхх пространств $\mathcal{H}_{k}, k \in \mathbb{Z}^{d}, k \neq 0$, определено представление $\mathfrak{D}_{0}(\mathcal{H}) \rightarrow \mathfrak{L}\left(\mathcal{H}_{k}\right)$ алгебры Ли $\mathfrak{D}_{0}(\mathcal{H})$. 
3.8. Случай $d=1$. Здесь индексы $i, j, k, \ldots \in \mathbb{Z}, \mathbb{Z}_{+}, \mathbb{N}$ (индекс $\mu$ опускаем за ненадобностью). В этом случае алгебра Ли $\mathfrak{s p}(2 ; \mathbb{F})=\mathfrak{s l}(2 ; \mathbb{F})$ и изоморфизм пункта (3) раздела 3.3 принимает вид

$$
\mathfrak{s l}(2 ; \mathbb{F}) \ni M=\left(\begin{array}{cc}
b & a \\
c & -b
\end{array}\right) \mapsto D=-a \frac{Q}{2}+b R+c \frac{P}{2} \in \mathfrak{D}^{2}(\mathcal{H})
$$

где $a, b, c \in \mathbb{F} . \mathrm{C}$ точностью до нормировки функционал Киллинга равен

$$
\phi(D, V)=a z+2 b y+c x, \quad D=-a \frac{Q}{2}+b R+c \frac{P}{2}, \quad V=-x \frac{Q}{2}+y R+z \frac{P}{2},
$$

а оператор Казимира -

$$
\Delta=R \circ R-\frac{1}{2}\{Q \circ P+P \circ Q\} .
$$

Подставляя сюда формулы пункта (1) раздела 3.3, получим

$$
\begin{aligned}
\Delta(f)= & 3\left(q \frac{\partial f}{\partial q}+\frac{\partial f}{\partial p} p\right)+\left(q^{2} \frac{\partial^{2} f}{\partial q^{2}}+2 q \frac{\partial^{2} f}{\partial q \partial p} p+\frac{\partial^{2} f}{\partial p^{2}} p^{2}\right)+ \\
& +2 \varkappa\left(2 \frac{\partial^{2} f}{\partial q \partial p}+q \frac{\partial^{3} f}{\partial q^{2} \partial p}+\frac{\partial^{3} f}{\partial q \partial p^{2}} p\right)+\varkappa^{2} \frac{\partial^{4} f}{\partial q^{2} \partial p^{2}}, \quad f \in \mathcal{H} .
\end{aligned}
$$

Рассмотрим в качестве примера уравнение $\Delta(f)=0$ в классе формальных степенных рядов (ср. с разделом 3.2 ),

$$
\Delta(f)=0, \quad f=\sum_{i, j=0}^{\infty} c_{i j} q^{i} p^{j}, \quad c_{i j} \in \mathbb{F}
$$

Для коэффициентов $c_{i j}$ получим систему уравнений

$$
\begin{aligned}
& (i+j)(i+j+2) c_{i j}+2 \varkappa(i+1)(j+1)(i+j+2) c_{i+1, j+1}+ \\
& +\varkappa^{2}(i+1)(j+1)(i+2)(j+2) c_{i+2, j+2}=0, \quad i, j \in \mathbb{Z}_{+} .
\end{aligned}
$$

Произведем замену индексов: $\left(i, j \in \mathbb{Z}_{+}\right) \leftrightarrow\left(k \in \mathbb{Z}, n \in \mathbb{Z}_{+}\right)$,

$$
\left\{\begin{array}{l}
i=n+k_{+}, \\
j=n+k_{-},
\end{array} \quad k_{+}=\left\{\begin{array}{ll}
k, & k \geqslant 0, \\
0, & k<0,
\end{array} \quad k_{-}= \begin{cases}0, & k \geqslant 0, \\
-k, & k<0\end{cases}\right.\right.
$$

так что $k=k_{+}-k_{-}, \quad|k|=k_{+}+k_{-}, \quad k=i-j, \quad n=(i+j-|i-j|) / 2$. Полагая $b_{k, n}=c_{i, j}$ и учитьвая, что $i+j=2 n+|k|, i j=n(n+|k|)$, для коэффициентов $b_{k, n}$ получаем рекуррентные соотношения

$$
b_{k, n+2}=-\frac{(|k|+2 n+2)}{\varkappa^{2}} \frac{2 \varkappa(n+1)(|k|+n+1) b_{k, n+1}+(|k|+2 n) b_{k, n}}{(n+1)(n+2)(|k|+n+1)(|k|+n+2)} .
$$


Последние имеют две серии решений: $g_{k n}$ и $h_{k n}$, где

$$
\begin{aligned}
& g_{k 0}=1, \quad g_{k 1}=0, \quad g_{k n}=\frac{(-1)^{n-1}(n-1)}{n ! \varkappa^{n}} \frac{|k|(|k|+2) \ldots(|k|+2 n-2)}{(|k|+1)(|k|+2) \ldots(|k|+n)} \\
& h_{k 0}=0, \quad h_{k 1}=1, \quad h_{k n}=\frac{(-1)^{n-1}}{(n-1) ! \varkappa^{n-1}} \frac{(|k|+2)(|k|+4) \ldots(|k|+2 n-2)}{(|k|+2)(|k|+3) \ldots(|k|+n)} .
\end{aligned}
$$

Следовательно,

(1) уравнение $\Delta(f)=0$ в классе формальных степенных рядов имеет базис линейно независимых решений:

$g_{k}(q, p)=\sum_{n=0}^{\infty} g_{k n} q^{n+k_{+}} p^{n+k_{-}}, \quad h_{k}(q, p)=\sum_{n=0}^{\infty} h_{k n} q^{n+k_{+}} p^{n+k_{-}}, \quad k \in \mathbb{Z}$,

коэффициенты $g_{k n}, h_{k n}$ определены выше.

\section{4. ЦЕНТРАЛЬНОЕ РАСШИРЕНИЕ АЛГЕБРЫ $\mathfrak{D}(\mathcal{H})$}

4.1. Дифференциальные формы. Для алгебры Гейзенберга $\mathcal{H}$ алгебра мультипликаторов $\mathfrak{M}(\mathcal{H})=\mathbb{F}$ (см. пункт (5) раздела 1.11 ), так что условие (i) из раздела 0.6 выполнено, а для данной алгебры $\mathcal{K}$ условия (ii)-(iv) сводятся к единственному условию: определен морфизм алгебр Ли $\psi: \mathfrak{D}(\mathcal{H}) \rightarrow \mathfrak{D}(\mathcal{K})$. Согласно разделу 0.6 годятся алгебры $\mathcal{H}, \mathbb{F}$ и $\mathfrak{D}(\mathcal{H})$.

Оставляя полное исследование дифференщиальных форм на будушее, рассмотрим простейший случай $\mathcal{K}=\mathfrak{M}(\mathcal{H})=\mathbb{F}$, когда

$$
\varphi=\operatorname{id}_{\mathbb{F}}: \mathfrak{M}(\mathcal{H})=\mathbb{F} \rightarrow \mathfrak{M}(\mathbb{F})=\mathbb{F}, \quad \text { a } \quad \psi=0: \mathfrak{D}(\mathcal{H}) \rightarrow \mathfrak{D}(\mathbb{F})=0
$$

Здесь, $\Omega^{*}(\mathcal{H} ; \mathbb{F})=\operatorname{Hom}_{\mathbb{F}}\left(\wedge^{*} \mathfrak{D}(\mathcal{H}) ; \mathbb{F}\right)=\left(\wedge^{*} \mathfrak{D}(\mathcal{H})\right)^{\prime}$. В частности, $\Omega^{0}(\mathcal{H} ; \mathbb{F})=\mathbb{F}$, а линейное пространство $\Omega^{1}(\mathcal{H} ; \mathbb{F})$, дуальное к линейному пространству $\mathfrak{D}(\mathcal{H})$, имеет базис $\left\{\theta_{i j} ;|i+j|>0\right\}$, дуальный к базису $\left\{R^{i j} ;|i+j|>0\right\}, \theta_{i j}\left(R^{k l}\right)=\delta_{i j}^{k l}$.

Согласно разделу 0.7 дифференциал $d=d_{u}: \Omega^{u}(\mathcal{H} ; \mathbb{F}) \rightarrow \Omega^{u+1}(\mathcal{H} ; \mathbb{F}), u \in \mathbb{Z}_{+}$, действует по правилу

$$
(d \omega)\left(D_{0}, \ldots, D_{u}\right)=\sum_{0 \leqslant v<w \leqslant u}(-1)^{v+w} \omega\left(\left[D_{v}, D_{w}\right], D_{0}, \ldots, \check{D}_{v}, \ldots, \check{D}_{w}, \ldots, D_{u}\right)
$$

для всех $\omega \in \Omega^{u}(\mathcal{H} ; \mathbb{F})$. В частности, $d \Omega^{0}(\mathcal{H} ; \mathbb{F})=d \mathbb{F}=0$, откуда $H^{0}(\mathcal{H} ; \mathbb{F})=$ ker $d_{0}=\mathbb{F}$. Легко также проверяется, что

$$
d \theta_{i j}=-\sum_{k, l, m, n} C_{i j}^{k l, m n} \theta_{k l} \cdot \theta_{m n}, \quad|i+j|>0
$$

Далее, с помошью равенства $[\mathfrak{D}(\mathcal{H}), \mathfrak{D}(\mathcal{H})]=\mathfrak{D}(\mathcal{H})($ см. пункт (1) раздела 2.4) выводим, что дифференциал $d \omega=0, \omega \in \Omega^{1}(\mathcal{H} ; \mathbb{F})$, тогда и только тогда, когда $\omega=0$, т.е. ker $d_{1}=0$. В частности, $H^{1}(\mathcal{H} ; \mathbb{F})=\operatorname{ker} d_{1} / \operatorname{im} d_{0}=0$. 
4.2. Алгебра Ли $\mathcal{H}$. Параллельно с алгеброй Ли $\mathfrak{D}(\mathcal{H})$ рассмотрим алгебру Ли $\mathcal{H}$ со скобкой $\{\cdot, \cdot\}$ (см. пункт (9) раздела 1.11 ). Напомним, что алгебра Ли $\mathcal{H}$ имеет базис $\rho^{i j}, i, j \in \mathbb{Z}_{+}^{d}$, и коммутационные соотношения

$$
\left\{\rho^{i j}, \rho^{k l}\right\}=\sum_{m, n} C_{m n}^{i j, k l} \rho^{m n}
$$

Как и в случае алгебры $\mathfrak{D}(\mathcal{H})($ см. пункт (1) раздела 2.4), коммутант $\{\mathcal{H}, \mathcal{H}\}=\mathcal{H}$. Заметим, что центр алгебры Ли $\mathcal{H}$ есть сеп $\mathcal{H}=\mathbb{F}$ (ср. с пунктом (3) раздела 1.11).

Пусть $\wedge^{*} \mathcal{H}$ - внешняя алгебра линейного пространства $\mathcal{H}$. Согласно обшей теории (см. [15]) и-коцепи алгебры Ли $\mathcal{H}$ со значениями в $\mathbb{F}$ определяются как линейные отображения из $\wedge^{u} \mathcal{H}$ в $\mathbb{F}, u \in \mathbb{Z}_{+}$, так что линейное пространство всех коцепей алгебры Ли $\mathcal{H}$ со значениями в $\mathbb{F}$ есть

$$
\mathfrak{C}^{*}(\mathcal{H} ; \mathbb{F})=\operatorname{Hom}_{\mathbb{F}}\left(\wedge^{*} \mathcal{H} ; \mathbb{F}\right)=\left(\wedge^{*} \mathcal{H}\right)^{\prime}
$$

В частности, $\mathfrak{C}^{0}(\mathcal{H} ; \mathbb{F})=\mathbb{F}$, линейное пространство $\mathfrak{C}^{1}(\mathcal{H} ; \mathbb{F})$, дуальное к $\mathcal{H}$, имеет дуальный базис $\vartheta_{i j}, \quad \vartheta_{i j}\left(\rho^{k l}\right)=\delta_{i j}^{k l}, \quad i, j, k, l \in \mathbb{Z}_{+}^{d}$. Дифференииал $d=d_{u}: \mathfrak{C}^{u}(\mathcal{H} ; \mathbb{F}) \rightarrow$ $\mathfrak{C}^{u+1}(\mathcal{H} ; \mathbb{F}), u \in \mathbb{Z}_{+}$, задается правилом

$$
(d \omega)\left(f_{0}, \ldots, f_{u}\right)=\sum_{0 \leqslant v<w \leqslant u}(-1)^{v+w} \omega\left(\left\{f_{v}, f_{w}\right\}, f_{0}, \ldots, \check{f}_{u}, \ldots, \check{f_{w}}, \ldots, f_{u}\right)
$$

для всех $\omega \in \mathfrak{C}^{u}(\mathcal{H} ; \mathbb{F})$. Здесь также $d_{u+1} \circ d_{u}=0$, так что определен комплекс Ли $\left\{\mathfrak{C}^{u}(\mathcal{H} ; \mathbb{F}), d_{u}, u \in \mathbb{Z}_{+}\right\}$с когомологиями $H_{\mathrm{Lie}}^{u}(\mathcal{H} ; \mathbb{F})=\operatorname{ker} d_{u} / \operatorname{im} d_{u-1}$.

По построению $d \mathfrak{C}^{0}(\mathcal{H} ; \mathbb{F})=d \mathbb{F}=0$, откуда $H_{\text {Lie }}^{0}(\mathcal{H} ; \mathbb{F})=\mathbb{F}$. Далее, в силу равенства $\{\mathcal{H}, \mathcal{H}\}=\mathcal{H}$, ker $d_{1}=0$, так что $H_{\text {Lie }}^{1}(\mathcal{H} ; \mathbb{F})=0$. Как и выше (см. (4.1)), легко проверяется равенство

$$
d \vartheta_{i j}=-\sum_{k, l, m, n} C_{i j}^{k l, m n} \vartheta_{k l} \cdot \vartheta_{m n}, \quad i, j \in \mathbb{Z}_{+}^{d} .
$$

4.3. Центральное расширение алгебры Ли $\mathfrak{D}(\mathcal{H})$. Видно, что алгебры Ли $\mathcal{H}$ и $\mathfrak{D}(\mathcal{H})$ очень похожи. Действительно, с помощью теоремы 2.4 легко проверить, что

(1) определена точная последовательность алгебр Ли

$$
0 \rightarrow \mathbb{F} \rightarrow \mathcal{H} \stackrel{\pi}{\rightarrow} \mathfrak{D}(\mathcal{H}) \rightarrow 0
$$

где $\mathbb{F}$ рассматривается как тривиальная коммутативная алгебра Ли, морфизм $\pi$ задается правилом: $\mathcal{H} \ni f \mapsto \pi(f)=\{f, \cdot\} \in \mathfrak{D}(\mathcal{H})$.

Другими словами, алгебра Ли $\mathcal{H}$ есть одномерное иентральное расширение алгебры Ли $\mathfrak{D}(\mathcal{H})$. Заметим, что $\pi\left(\rho^{00}\right)=0, \pi\left(\rho^{i j}\right)=R^{i j}, i, j \in \mathbb{Z}_{+}^{d},|i+j|>0$.

Дуальная последовательность имеет вид

$$
0 \longleftarrow \mathbb{F} \longleftarrow \mathfrak{C}^{1}(\mathcal{H} ; \mathbb{F}) \stackrel{\pi^{*}}{\longleftarrow} \Omega^{1}(\mathcal{H} ; \mathbb{F}) \longleftarrow 0
$$


где $\pi^{*}\left(\theta_{i j}\right)=\vartheta_{i j}, i, j \in \mathbb{Z}_{+}^{d},|i+j|>0$.

Согласно общей теории когомологий алгебр Ли (см. [15]), каждое одномерное центральное расширение алгебры Ли ассоциировано с некоторой замкнутой 2-формой на этой алгебре. В нашем случае имеется разложение $\mathcal{H}=\mathbb{F} \cdot 1+\mathcal{H}_{\star}$ (см. раздел 2.3$)$, и искомая форма $\chi \in \Omega^{2}(\mathcal{H} ; \mathbb{F})$ определяется условием

$$
\chi(\pi(f), \pi(g))=\operatorname{pr}_{1}(\{f, g\}), \quad f, g \in \mathcal{H},
$$

где проекция $\operatorname{pr}_{1}: \mathcal{H} \rightarrow \mathbb{F}$. Подробнее, $\operatorname{pr}_{1}(f)=f(0)=f_{00}=\vartheta_{00}(f)$ для всякого $f=$ $\sum_{i, j} f_{i j} \rho^{i j} \in \mathcal{H}$. Следовательно, $\operatorname{pr}_{1}(\{f, g\})=\vartheta_{00}(\{f, g\})=-\left(d \vartheta_{00}\right)(f, g)$, так что определяюшее условие принимает вид $\chi(\pi(f), \pi(g))=-\left(d \vartheta_{00}\right)(f, g)$. Учитывая равенство (4.2), формулу

$$
C_{00}^{i j, k l}=\sum_{|r|>0} \varkappa^{|r|-1} r !\left(\delta_{0}^{i} \delta_{r}^{j} \delta_{r}^{k} \delta_{0}^{l}-\delta_{r}^{i} \delta_{0}^{j} \delta_{0}^{k} \delta_{r}^{l}\right),
$$

соотношение пункта (4) раздела 0.8 и равенства типа $\vartheta_{i j}(f)=f_{i j}=\theta_{i j}(\pi(f))$, окончательно получаем, что

(2) одномерное центральное расширение (4.3) ассоциировано с 2-формой

$$
\chi=2 \sum_{|r|>0} \varkappa^{|r|-1} r ! \theta_{0 r} \cdot \theta_{r 0} \in \Omega^{2}(\mathcal{H} ; \mathbb{F}) .
$$

Обратимся к свойствам формы $\chi$. По построению эта форма замкнутая. Проверим это свойство непосредственно: пусть $f_{1}, f_{2}, f_{3} \in \mathcal{H}$, тогда

$$
\begin{aligned}
(d \chi)\left(\pi\left(f_{1}\right), \pi\left(f_{2}\right), \pi\left(f_{3}\right)\right)= & -\chi\left(\left[\pi\left(f_{1}\right), \pi\left(f_{2}\right)\right], \pi\left(f_{3}\right)\right)- \\
& -\chi\left(\left[\pi\left(f_{2}\right), \pi\left(f_{3}\right)\right], \pi\left(f_{1}\right)\right)-\chi\left(\left[\pi\left(f_{3}\right), \pi\left(f_{1}\right)\right], \pi\left(f_{2}\right)\right)= \\
= & -\vartheta_{00}\left(\left\{\left\{f_{1}, f_{2}\right\}, f_{3}\right\}+\left\{\left\{f_{2}, f_{3}\right\}, f_{1}\right\}+\left\{\left\{f_{3}, f_{1}\right\}, f_{2}\right\}\right)=0,
\end{aligned}
$$

где мы использовали равенство $[\pi(f), \pi(g)]=\pi(\{f, g\}), f, g \in \mathcal{H}$, (см. пункт (1) раздела 4.3$)$ и тождество Якоби.

Однако форма $\chi$ не является точной. Действительно, предположим, что $\chi=d \Psi \mathrm{c}$ некоторой 1-формой

$$
\Psi=\sum_{|i+j|>0} \Psi^{i j} \theta_{i j} \in \Omega^{1}(\mathcal{H} ; \mathbb{F}),
$$

коэффициенты $\Psi^{i j} \in \mathbb{F}$. Тогда для всех $f, g \in \mathcal{H}$ будем иметь

$$
\begin{aligned}
\chi(\pi(f), \pi(g)) & =(d \Psi)(\pi(g), \pi(g))=-\Psi([\pi(f), \pi(g)])=-\Psi(\pi(\{f, g\}))= \\
& =-\psi(\{f, g\})=(d \psi)(f, g),
\end{aligned}
$$


где

$$
\psi=\pi^{*} \Psi=\sum_{|i+j|>0} \Psi^{i j} \vartheta_{i j} \in \mathfrak{C}^{1}(\mathcal{H} ; \mathbb{F}) .
$$

С другой стороны, по построению $\chi(\pi(f), \pi(g))=-\left(d \vartheta_{00}\right)(f, g)$. Итак, $d\left(\psi+\vartheta_{00}\right)=0$, и, значит, $\psi+\vartheta_{00}=0$, поскольку ker $d_{1}=0$ (см. раздел 4.2). Подробнее,

$$
\sum_{|i+j|>0} \Psi^{i j} \vartheta_{i j}+\vartheta_{00}=0
$$

что, очевидно, невозможно. Полученное противоречие показывает, что $\chi$ не является точной.

Таким образом, доказано

4.4. ПРЕДЛОЖЕНИЕ. Форма

$$
\chi=2 \sum_{|r|>0} \varkappa^{|r|-1} r ! \theta_{0 r} \cdot \theta_{r 0}
$$

задает нетривиальный әлемент $[\chi] \in H^{2}(\mathcal{H} ; \mathbb{F})$.

В частности, центральное расширение $(4.3)$ нетривиальное, и $H^{2}(\mathcal{H} ; \mathbb{F}) \neq 0$.

4.5. Алгебра инвариантности формы $\chi$. Форма $\omega \in \Omega^{*}(\mathcal{A} ; \mathcal{K})$ называется инвариантной относительно дифференцирования $D \in \mathfrak{D}(\mathcal{A})$ (мы используем обозначения раздела 0.7 ), если $L_{D} \omega=0$. В силу равенства пункта (2) раздела 0.7 множество всех дифференцирований, относительно которых $\omega$ инвариантна, образует подалгебру (алгебру инвариантности $\omega$ ) алгебры Ли $\mathfrak{D}(\mathcal{A})$.

Вычислим алгебру инвариантности формы $\chi$. Пусть

$$
F=\sum_{|i+j|>0} f_{i j} R^{i j}
$$

тогда в силу пункта (4) раздела $0.7 L_{F} \chi=\iota_{F}(d \chi)+d\left(\iota_{F} \chi\right)=d\left(\iota_{F} \chi\right)$, где мы учли, что $d \chi=0$. Форма $\iota_{F} \chi \in \Omega^{1}(\mathcal{H} ; \mathbb{F})$ и $\operatorname{ker} d_{1}=0$ (см. раздел 4.1), так что $L_{F} \chi=0$ тогда и только тогда, когда $\iota_{F} \chi=0$. Подробнее последнее условие записывается в виде

$$
\chi(F, G)=\sum_{|r|>0} \varkappa^{|r|-1} r !\left(f_{0 r} g_{r 0}-f_{r 0} g_{0 r}\right), \quad \forall G=\sum_{|k+l|>0} g_{k l} R^{k l} \in \mathfrak{D}(\mathcal{H}),
$$

и имеет очевидное решение $f_{0 r}=f_{r 0}=0$ при $|r|>0$, остальные коэффициенты $f_{i j}$ произвольные. Итак,

(1) алгебра инвариантности формы $\chi$ состоит из всех дифференширований вида

$$
F=\sum_{|i|>0,|j|>0} f_{i j} R^{i j} \in \mathfrak{D}(\mathcal{H})
$$

Благодарности. Работа выполнена при поддержке гранта РФФИ 98-01-00640. 


\section{Список литературы}

[1] Дж. Макки. Лекции по математическим основам квантовой механики. М.: Мир, 1965.

[2] Л. Д. Фаддеев, О.А. Яћубовский. Лекции по квантовой механике для студентов математиков. Л.: Изд-во ЛГУ, 1980.

[3] N. Fleury, A. Turbiner. On polynomial relations in the Heisenberg algebra. Preprint, funct-an/9403002, 1994.

[4] A. Turbiner. Invariant identities in the Heisenberg algebra. Preprint, hep-th/9410128, 1998.

[5] M. Pillin. On the deformability of Heisenberg algebras. Preprint, q-alg/9508014, 1995.

[6] M. Irac-Astaud. A three-parameter deformation of the Weyl-Heisenberg algebra: differential calculus and invariance. Preprint, q-alg/9609008, 1996.

[7] B. Abdesselam. The twisted Heisenberg algebra. Preprint, q-alg/9610021, 1996.

[8] M.S. Plyushchay. R-deformed Heisenberg algebra. Preprint, hep-th/9701065, 1997.

[9] A. Connes. Noncommutative geometry. New York: Academic Press, 1994.

[10] J. Madore. An introduction to noncommutative differential geometry and its physical applications. Cambridge: Cambridge University Press, 1995.

[11] M. Dubois-Violette, R. Kerner, J. Madore. J. Math. Phys. 1990. V. 31. № 2. P. 316.

[12] M. Dubois-Violette, P. W. Michor. J. Geom. Phys. 1996. V. 20. P. 218.

[13] T. Masson. J. Math. Phys. 1996. V. 37. № 5. P. 2484.

[14] H.-D. Cao, J. Zhou. On quantum de Rham cohomology. Preprint, DG/9806157, 1998.

[15] Д. Б. Фукс. Когомологии бесконечномерных алгебр Ли. М.: Наука, 1984.

[16] V. V. Zharinov. Integral transforms and special functions. 1998. V. 7. № 1-2. P. 155.

[17] E. R. Kolchin. Differential algebra and algebraic groups. New York: Academic Press, 1950.

[18] I. Kaplansky. An introduction to differential algebra. Paris: Hermann, 1957.

[19] H. Х. Ибрагимов. Группы преобразований в математической физике. М.: Наука, 1983.

[20] V. V. Zharinov. Lecture notes on geometrical aspects of partial differential equations. Singapore: World Scientific, 1992.

[21] С. Маклейн. Гомология. М.: Мир, 1966.

[22] М. А. Наймарк. Теория представлений групп. М.: Наука, 1976.

[23] Д. П. ЖКелобенко. Лекции по теории групп Ли. Дубна: ОИЯИ, 1965.

Поступила в редакцию 21.VII.1998 г. 